\title{
Two-Dimensional Linear Prediction and Its Application to Adaptive Predictive Coding of Images
}

\author{
PETROS A. MARAGOS, STUdENT MEMBER, IEEE, RONALD W. SCHAFER, FELLOW, IEEE, AND \\ RUSSELL M. MERSEREAU, FELLOW, IEEE
}

\begin{abstract}
This paper summarizes a study on two-dimensional linear prediction of images and its application to adaptive predictive coding of monochrome images. The study was focused on three major areas: two-dimensional linear prediction of images and its performance, implementation of an adaptive predictor and adaptive quantizer for use in image coding, and linear prediction and adaptive predictive coding of density (logarithm of intensity) images.

Among the issues investigated are: autoregressive modeling of 2-D image sequences, estimation of the nonzero average bias of the image samples, stability of the inverse prediction error filter, and estimation of the parameters of a 2-D separable linear predictor. The implementation of the adap tive predictor is based on the results of linear predictive analysis. The adaptive quantization of the prediction error signal is done by using a flexible three-level quantizer for code words of fixed or variable length. The above ideas are further applied to density images for exploiting the multiplicative structure of images.

The results of this research indicate that by using adaptive prediction and quantization, intensity and density coded images of high quality can be obtained at information rates as low as 0.7 bits/pixel.
\end{abstract}

\section{INTRODUCTION}

$\mathrm{T}$ HE techniques of linear prediction have been applied with great success in many problems of speech processing [1] [4]. Linear prediction is established as the predominant technique for extracting speech parameters and for speech coding at low bit rates [5]. This success in processing speech signals suggests that similar techniques might be useful in modeling and coding of 2-D image signals. Due to the extensive computation required for its implementation in two dimensions, only the simplest forms of linear prediction have received much attention in image coding [6], [7]. However, current reductions in cost and increases in speed of digital signal processing hardware suggest that it is no longer necessary to limit our attention to simple processing schemes for image modeling and coding. Thus, this paper consists of two parts. The first part is concerned with autoregressive modeling of 2-D image signals, and the use of two-dimensional linear predictive analysis for extracting the parameters of this model. The second part reports the performance of an adaptive predictive image coding scheme which uses adaptive two-dimensional linear prediction and an adaptive three-level quantizer to quantize the prediction error signal at low bit rates.

Manuscript received September 1, 1983. This work was supported by the Joint Services Electronics Program of the Department of Defense under Contract DAAG29-81-K-0024.

The authors are with the School of Electrical Engineering, Georgia Institute of Technology, Atlanta, GA 30332.

\section{2-D Linear Prediction}

\section{A. Image Model}

Various autoregressive image models have been examined by different researchers [8] aiming at different goals. Our objective is to introduce an autoregressive model which will account for the spatial variability of image sequences and for the fact that intensity image samples possess a nonzero average bias since they always assume nonnegative values. Hence, let us consider the image model in Fig. 1(a), where $x(m, n)$ denotes the 2-D sequence of intensity image samples and $a_{0}$ represents a locally constant bias coefficient added to the input of the feedback system. This feedback system, which accounts for the autoregressive nature of our model, is called the predictor, and its corresponding transfer function is

$$
P\left(z_{1}, z_{2}\right)=\sum_{\substack{k \\(k, l) \in \Pi}} \sum_{\substack{l \\(k,}} a(k, l) z_{1}^{-k} z_{2}^{-l}
$$

where $a(k, l)$ is a 2-D prediction coefficient array and $\Pi$ is a set of integer pairs to be specified later. Fig. 1(a) implies the following difference equation relating the output $x(m, n)$ and input $u(m, n)$ :

$$
x(m, n)=\sum_{\substack{k \\(k, l) \in \Pi}} \sum_{l} a(k, l) x(m-k, n-l)+a_{0}+u(m, n) .
$$

The 2-D input sequence $u(m, n)$ may be thought of as either a zero mean white noise field or as a 2-D unit impulse, depending upon whether we view the problem from a stochastic or from a deterministic point of view.

An equivalent image model could result if we think of the 2-D sequence $x(m, n)$ as being the sum of a zero mean autoregressive sequence $y(m, n)$ and a locally constant dc offset $B$. Then, as Fig. 1(b) implies,

$$
\begin{aligned}
x(m, n)=y(m, n)+B= & \sum_{\substack{k \\
(k, l) \in \Pi}} a(k, l) y(m-k, n-l) \\
& +B+u(m, n) .
\end{aligned}
$$

Comparing the equivalent difference equations (2) and (3) we can find a relation between $a_{0}$ and $B$

$$
a_{0}=B\left[1-\sum_{\substack{k \\(k, l) \in \mathrm{m}}} \sum_{\substack{l \\(k, l)}} a=B[1-P(1,1)] .\right.
$$




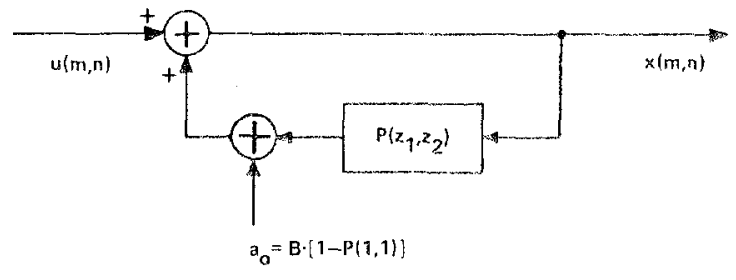

(a)

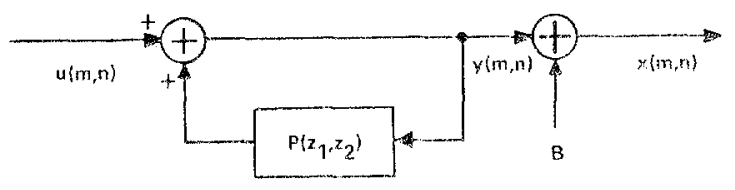

(b)

Fig. 1. Autoregressive image models. (a) Bias at the input. (b) Bias at the output.

The bias coefficient $a_{0}$ can be thought of as a bias at the input, whereas $B$ represents a bias at the output. In both cases, the inclusion of a bias parameter accounts for the fact that the intensity image samples $x(m, n)$ are explicitly biased, since they are always nonnegative. The advantage of (2) containing $a_{0}$ is the linearity of the normal equations which are involved in the estimation of the parameters of the model. The difference equation (2) represents either a means for synthesizing the image signal $x(m, n)$ if we know the model coefficients and the excitation $u(m, n)$, or a means for extracting the model parameters if we have available the signal $x(m, n)$ and make some assumptions about the input $u(m, n)$.

The set II of integer pairs spanned by the indexes $(k, l)$ of the prediction coefficient array $a(k, l)$ is called the region of support of the predictor or the prediction mask. This set determines the spatial causality of the model. Spatial causality is not inherent in image formation. However, it may be imposed by the scanning mechanism for a raster of image samples. Our ultimate objective is to use the optimal estimates of the model coefficients for resynthesizing the image signal $x(m, n)$ at the decoder of the image coding scheme. Therefore, (2) must be recursively computable. This limits the possible prediction masks [9] only to causal, nonsymmetric half-plane masks. Sacrificing some generality, we have limited our study to causal prediction masks which possess a $Q \times Q$ quarter-plane region of support; namely masks where $(k, l)$ range over all integer pairs in the set

$$
\mathrm{II}=\{(k, l): 0 \leqslant k, l \leqslant Q-1 \text { and }(k, l) \neq(0,0)\} .
$$

The number of prediction coefficients included in a $Q \times Q$ quarter-plane prediction mask, also called predictor order, is

$$
P=Q^{2}-1 \text {. }
$$

\section{B. 2-D Linear Prediction of Intensity Images}

The parameters of the image model $\left(a(k, l)\right.$ and $\left.a_{0}\right)$ can be estimated by the method of linear predictive analysis in which it is assumed that the model coefficients are those which minimize the mean-squared value of the $2 \mathrm{D}$ prediction error sequence

$$
e(m, n)=x(m, n)-\sum_{\substack{k \\(k, l) \in \Pi}} a(k, l) x(m-k, n-l)-a_{0} .
$$

The mean-squared prediction exror residual is defined as

$$
E=\sum_{m=L}^{U} \sum_{n=L}^{U} e^{2}(m, n)
$$

where the limits $L, U$ will be specified later. The prediction error filter is a linear system with corresponding transfer function

$$
A\left(z_{1}, z_{2}\right)=1-P\left(z_{1}, z_{2}\right)
$$

where $P\left(z_{1}, z_{2}\right)$ is defined in (1). The optimal model coefficients are those which minimize $E$, and consequently they satisfy the normal equations

$$
\begin{aligned}
& \sum_{\substack{k \\
(k, l) \in \Pi}} \sum_{l} a(k, l) \phi(k, l: i, j)+a_{0} S(i, i) \\
& \quad=\phi(0,0: i, j), \quad(i, j) \in \Pi \\
& \sum_{\substack{k \\
(k, l) \in \Pi}} \sum_{l} a(k, l) S(k, l)+a_{0} N_{s}=S(0,0)
\end{aligned}
$$

where

$$
\begin{gathered}
\phi(k, l: i, j)=\sum_{m=L}^{U} \sum_{n=L}^{U} x(m-k, n-l) x(m-i, n-j) \\
S(k, l)=\sum_{m=L}^{U} \sum_{n=L}^{U} x(m-k, n-l)
\end{gathered}
$$

and $N_{s}$ is the number of samples in the region of support of the 2-D sequence $e(m, n)$. In (11) $m$ and $n$ range over a set of integers corresponding to a particular $M \times M$ region of the image, called the analysis frame. Over each analysis frame we assume that the model coefficients are fixed, and we compensate for the nonstationarity of the image by using small analysis frames and computing a different model for each frame.

The minimum prediction error residual can be shown to be given by

$$
\begin{aligned}
E_{\min }= & \phi(0,0: 0,0)-\sum_{\substack{k \\
(k, l) \in \Pi}} a(k, l) \phi(0,0: k, l) \\
& -a_{0} S(0,0) .
\end{aligned}
$$

In order to adopt a matrix representation for $(10)$, a onedimensional indexing [9] of the prediction coefficient array $a(k, l)$ is defined as follows:

$$
I(k, l)=i=l \cdot Q+k .
$$

The above indexing corresponds to a rowwise scanning of $a(k, l)$, and it is not the only possible one. For $(k, l) \in \Pi \cup$ $(0,0)$ the index $i=I(k, l)$ ranges over the integers $0 \leqslant i \leqslant P$ in a one-to-one relationship with the integer pair $(k, l)$. Thus, we can recover the integer pair $(k, l)=I^{-1}(i)$, and the four-index 
dependence of $\phi(k, l: i, j)$ can be written as a two-index dependence by writing $\phi(k, l: i, j)=\phi(q: r)$, where $q=I(k, l)$ and $r=$ $I(i, j)$. Similarly, $S(k, l)=S(q)$. At this point, (10) can be rewritten in matrix form as

$$
C \alpha=c
$$

where

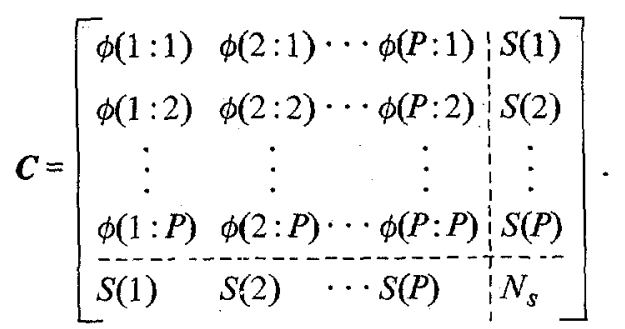

For reasons explained later, we denote by $\boldsymbol{R}$ the upper left $P \times$ $P$ principal submatrix of $C$ which contains only correlation lags as entries, and

$$
\begin{aligned}
\boldsymbol{\alpha} & =\left[a\left(I^{-1}(1)\right), \cdots, a\left(I^{-1}(P)\right) !, a_{0}\right]^{T} \\
& =\left[a^{T}, a_{0}\right]^{T} \\
c & =[\phi(0: 1), \cdots, \phi(0: P) !, S(0)]^{T} \\
& =\left[\boldsymbol{r}^{T}, S(0)\right]^{T}
\end{aligned}
$$

where $[\cdot]^{T}$ denotes the transpose of a vector. The $(P \times P)$ matrix $R$ and the $(P \times 1)$ vectors $a$ and $r$ are called, respectively, the correlation matrix, the prediction coefficient vector, and the correlation vector.

Bias Estimation: There are several issues to be considered in the computation of the model coefficients. One concern is the way the bias $B$ is estimated. The derivation of the normal equations in (10) assumed that the bias remains constant over each analysis frame. There are three ways to handle the bias: 1) Estimate $a_{0}$ by solving the $(P+1) \times(P+1)$ system $\boldsymbol{C \alpha}=\boldsymbol{c}$, and then exploit the relation (4) between $a_{0}$ and $B$. This approach is henceforth denoted by TBLP (true bias linear prediction). 2) Estimate the local mean of the signal over the $M \times M$ frame as being an approximation to $B$, subtract it from $x(m, n)$ and then use only (10a) with $a_{0}=0$, solving a $(P \times P)$ system. We denote this approach as LMLP (local mean linear prediction). 3) Do not subtract any estimate of the bias $B$ and use only (10a) with $a_{0}=0$; i.e., solve only the $(P \times P)$ system $\boldsymbol{R a}=\boldsymbol{r}$. This last approach is simply denoted LP (linear prediction).

Covariance Versus Autocorrelation: Another concern is the determination of the range of summation in (8) and (11). One approach is to limit the summation to the $M \times M$ analysis frame. This implies setting $L=0$ and $U=M-1$ in (8), (11), and $N_{s}=M^{2}$ in (10b), and it results in bringing inside the analysis frame samples from the borders of the frame to be supplied as needed in the computation of (11). We call this the "covariance method" as in one-dimensional linear predictive analysis [5]. Since $\phi(k, l: i, j)=\phi(i, j: k, l)$ [see (11a)], the matrix $C$ in (14) is symmetric. Moreover, $C$ is almost always positive-definite, except for some degenerative cases where it is positive-semidefinite. Therefore, it can be inverted using Cholesky decomposition [5] .

Another approach is the so-called "autocorrelation method."
In this case the signal $x(m, n)$ is assumed to be zero everywhere outside the $M \times M$ frame and the summation for the squared error $E$ involves all the nonzero values of $e(m, n)$; i.e., $L=0$ and $U=M+Q-2$ in (8) where $Q \times Q$ is the size of the prediction mask. Therefore, we must set $N_{s}=(M+Q-1)^{2}$ in $(10 \mathrm{~b})$. In the autocorrelation case it can be proven easily that the correlation lags $\phi(k, l: i, j)$ possess the following properties:

$$
\phi(k, l: i, j)=\left\{\begin{array}{r}
\phi(0,0:|k-i|,|l-j|), \\
\text { if }(k-i)(l-j) \geqslant 0 \\
\phi(0,0:|k-i|,-|l-j|), \\
\text { if }(k-i)(l-j)<0 .
\end{array}\right.
$$

Therefore, we can replace $\phi(k, l: i, j)$ with $R(|k-i|, \pm|l-j|)$ where

$$
\begin{aligned}
R(k, l) \triangleq & \sum_{m=\max (0, k)}^{\min (M-1, k+M-1) \min (M-1, l+M-1)} \sum_{n=\max }(0, l) \\
& \cdot x(m, n) x(m-k, n-l) .
\end{aligned}
$$

From (17), (18) it can be shown that $R(k, l)=R(-k ;-l)$. Also, in the autocorrelation method the sums $S(k, l)$ defined in (11b) assume the same value for all different lags $(k, l)$. Therefore, the $P+1$ equations of the system (10) are decoupled in a system of $P$ equations in $P$ unknowns, plus an extra decoupled equation for $a_{0}$ in the TBLP case. More precisely, the equations in (10) now take the form

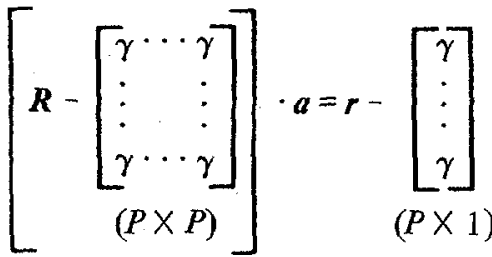

$$
\begin{aligned}
& a_{0}=B\left[1-\sum_{\substack{k \\
(k, l) \in \Pi}} a(k, l)\right]
\end{aligned}
$$

where $\boldsymbol{R}, \boldsymbol{a}$, and $\boldsymbol{r}$ are defined in (14), (15), and (16), respectively, and $\gamma, B$ are known constants

$$
\gamma=B \cdot\left[2 \cdot S(0,0)-B \cdot N_{s}\right]
$$

with

$$
B=\left\{\begin{array}{lll}
S(0,0) / N_{s}, & \text { for } & \text { TBLP } \\
S(0,0) / M^{2}, & \text { for } & \text { LMLP } \\
0, & \text { for } & \text { LP. }
\end{array}\right.
$$

Thus, in the autocorrelation method the optimum bias $B$ will always be a little smaller than the local mean, and the system of normal equations to be solved will always consist of $P$ equations regardless of the way the bias is handled.

The matrix of the system of equations in (19a) is a $P \times P$ symmetric block Toeplitz matrix which is always positivedefinite. There exist methods for the inversion of such matrices which are more efficient than the Cholesky decomposition [10]. The storage requirements for the autocorrelation method, both for the signal $x(m, n)$ and for the entries of the correlation matrix, are fewer than in the covariance method. 
In addition, by comparing (11a) and (18) we can infer that the covariance method requires a greater number of multiplications and additions for the computation of each correlation lag. In the one-dimensional case, if the autocorrelation method is used, the stability of the inverse prediction error filter is guaranteed, but with the covariance method it is not. In the two-dimensional case neither approach can guarantee stability, as shown in [11].

Prediction Error: To examine the performance of twodimensional linear prediction, we computed the total normalized prediction error by partitioning the image into an integer number of nonoverlapping frames, summing the mean-squared prediction error (8) from each individual frame, and dividing the total error by the energy of the image signal $x(m, n)$ over the whole image. All the following results refer to the "Girl" image of Fig. 2(a), but similar results were obtained for other images as well.

Table I shows a comparison among the different approaches for handling the bias using the covariance method and various predictor orders and frame sizes. In the column headings of Table I, the first digit $P$ refers to the predictor order and $M$ refers to the size of the $M \times M$ analysis frame. Although the results give a slight superiority to the TBLP method, all three methods yield comparable prediction errors. This is not unexpected since the available test images were oversampled, and thus there was enough correlation between samples for all of the methods to work satisfactorily. However, as shown later, we found a significant difference in the stability of the resulting models.

A prediction error image is shown in Fig. 2(b), where the prediction error samples (which are both positive and negative) were mapped linearly onto the range 1-256 (8 bits/pixel) so that the prediction error sequence $e(m, n)$ could be displayed as an image. It is obvious that linear prediction removes much of the redundant information from the image, leaving only information about the edges.

Perspective plots of the magnitude of the 2-D Fourier transform of the original image and its prediction error are illustrated in Fig. 3(a) and (b). We see that in the frequency domain the linear prediction flattens the original low-pass image spectrum.

Figs. 4 and 5 provide us with an informative comparison between the performance of the covariance and autocorrelation method. In both figures the ordinate gives the mean-squared prediction error (per frame, using the TBLP method) normalized by the energy of the image signal $x(m, n)$ over each analysis frame, and averaged over 64 frames uniformly distributed over the whole image of Fig. 2(a). In Fig. 4 the variation of the error versus the size $M$ of the $M \times M$ frame is illustrated. The covariance method is shown to give a consistently smaller prediction error and to be almost insensitive to frame size variations for a fixed predictor order $P=3$. The error resulting from the autocorrelation method is reduced significantly by increasing the frame size, which implies that for large frames both methods yield identical results. Fig. 5 shows the variation of the error versus the size $Q$ of the $Q \times Q$ prediction mask for a fixed frame size $M=32$. Again the covariance method performs better than the autocorrelation method. The size of the prediction mask has little effect upon the per-

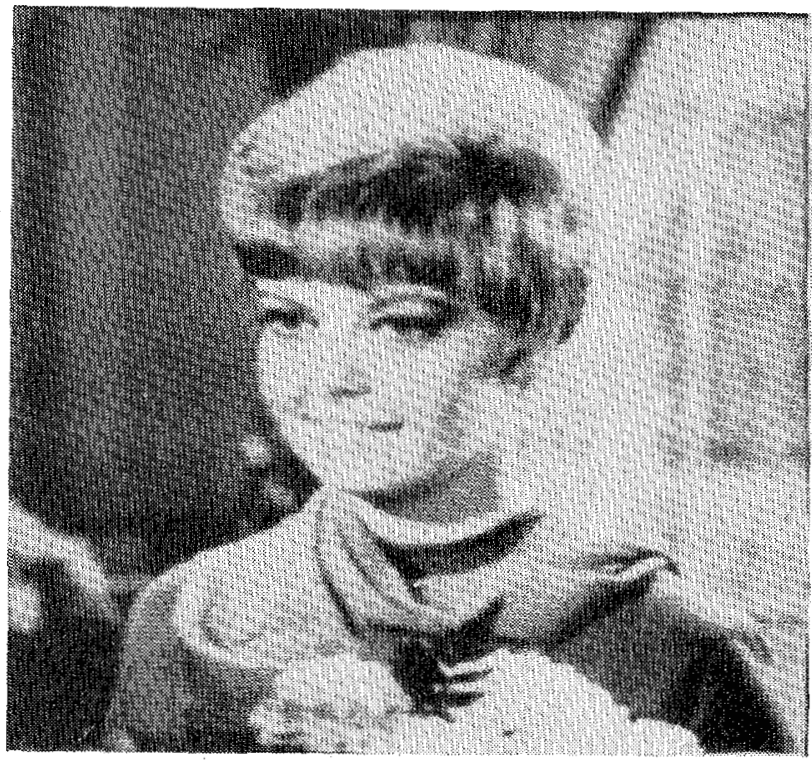

(a)

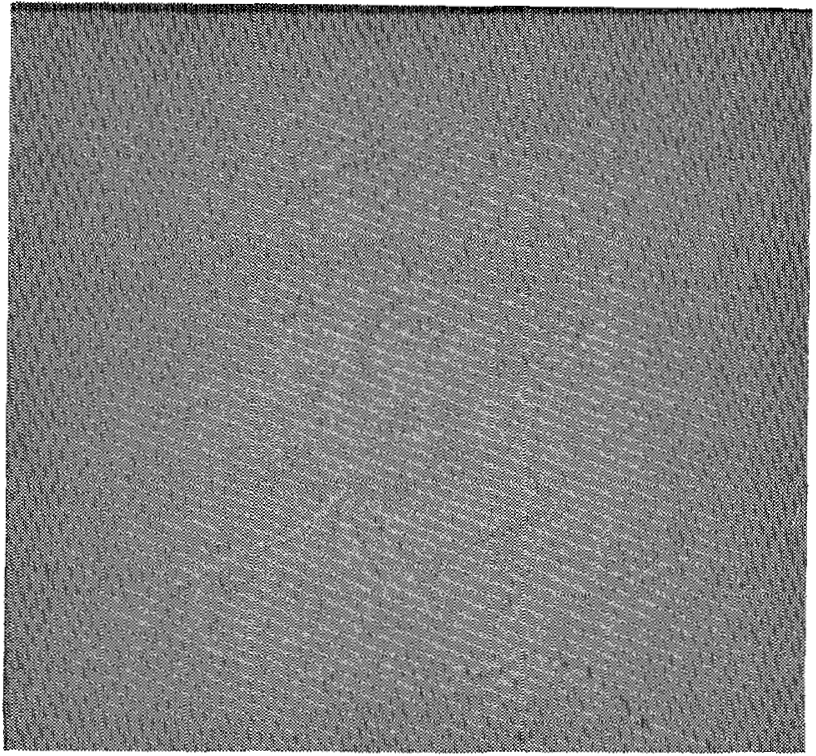

(b)

Fig. 2. (a) Original 8 bit/pixel image $(256 \times 256$ pixels $)$. (b) Prediction error image $(P=8, M=32)$.

TABLE I

Total Normalized Prediction Error (Percent) for Linear Prediction of Intensity Images

\begin{tabular}{cccc}
\hline Method/Conditions $(P, M)$ & 3,32 & 3,16 & 8,32 \\
\hline TBLP & 0.707 & 0.643 & 0.622 \\
LMLP & 0.710 & 0.655 & 0.624 \\
LP & 0.715 & 0.669 & 0.633 \\
\hline
\end{tabular}

formance of either method for this image. Prediction masks bigger than $3 \times 3$ or $4 \times 4$ coefficients do not significantly improve the prediction error.

\section{Predictor Stability}

Another very important consideration concerning the allpole image model of Fig. 1 is its stability, because the model is a fundamental component of both the coder and the decoder in an adaptive predictive coding system [1], [2] and instabili- 


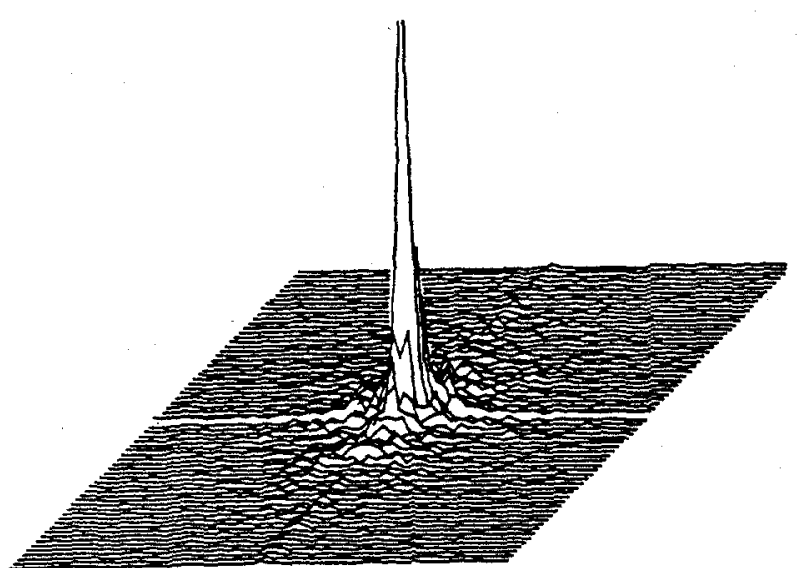

(a)

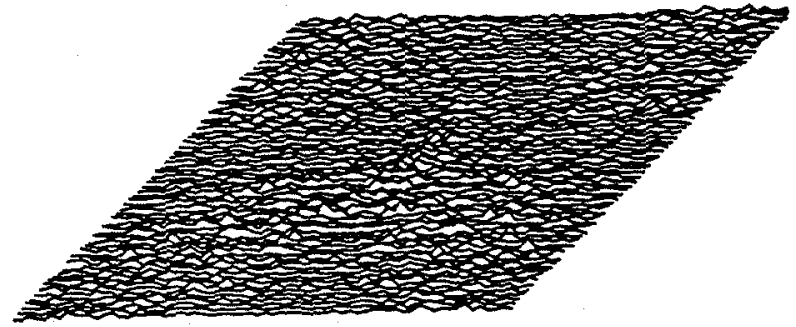

(b)

Fig. 3. Perspective plots of the magnitude of the 2-D Fourier transform (a) of the original image, and (b) of the prediction error signal $(P=8, M=32$ ) (the prediction error is magnified three times relative to the original image).

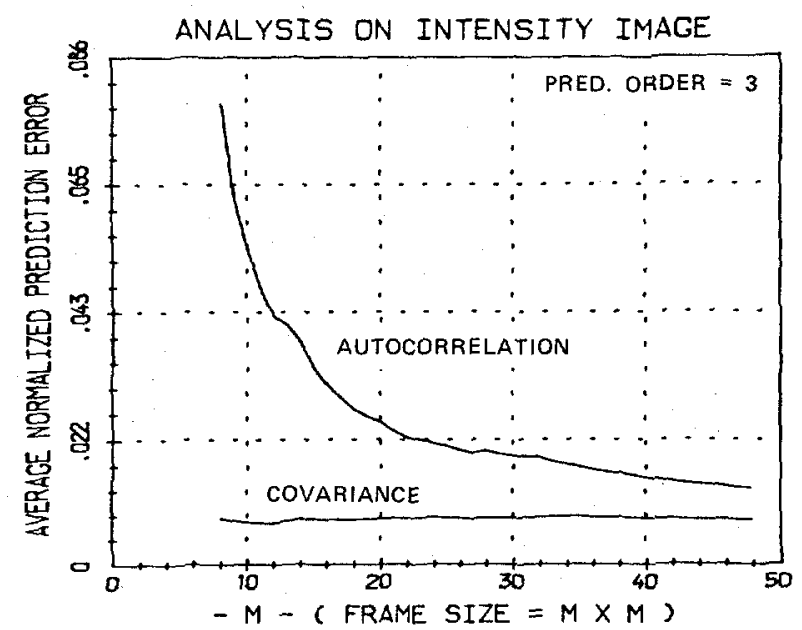

Fig. 4. Variation of prediction error versus frame size for intensity images $(P=3)$.

ties could lead to large errors upon reconstructing the image signal.

The system, about whose stability we must be concerned, is the inverse prediction error filter. Its transfer function is $1 / A\left(z_{1}, z_{2}\right)$, where $A\left(z_{1}, z_{2}\right)$ is given by (9). The impulse response of $A\left(z_{1}, z_{2}\right)$ has support only on the first quadrant, because we used a quarter-plane prediction mask. Therefore, the inverse prediction error filter is recursively computable, and conditions for its stability can be found in Huang's theorem [12], from which we can derive the following necessary condition.

Theorem: A necessary condition for the stability of the first quadrant recursive filter $1 / A\left(z_{1}, z_{2}\right)$ is

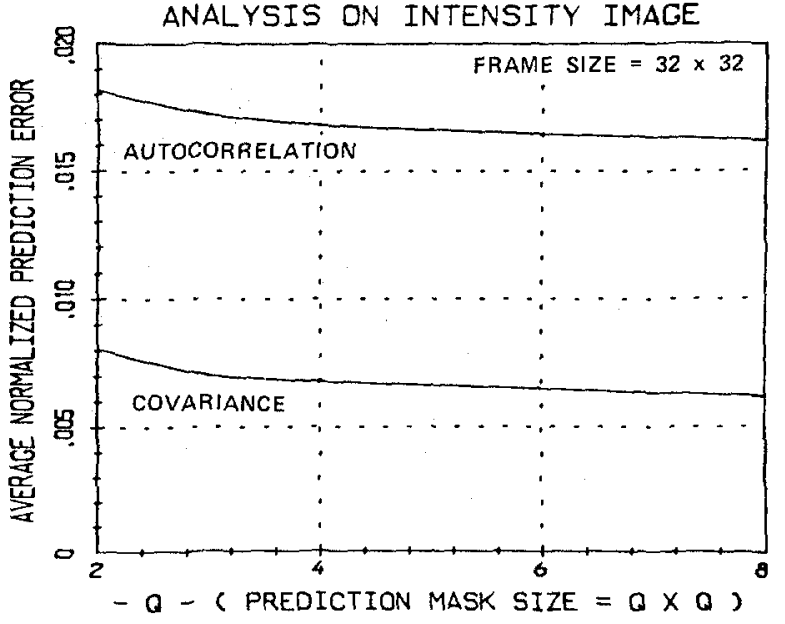

Fig. 5. Variation of prediction error versus size of prediction mask for intensity images $(M=32)$.

$$
A(1,1)=1-\sum_{\substack{k \\(k, l) \in \mathrm{I}}} \sum_{l} a(k, l)>0 .
$$

The proof for the above condition is given in the Appendix. Let us now recall that $P(1,1)=1-A(1,1)$. If $P(1,1) \geqslant 1$, we conclude from (21) that the model is necessarily unstable. If $P(1,1)<1$, then the predictor might be stable since its coefficients are away from the point of marginal instability: $P(1,1)=1$. Also recall from (4) that $a_{0}=B \cdot[1-P(1,1)]$. Thus, the bias interacts with the stability of the model. For positive image signals, the bias $B$ must be a positive number. Thus, comparing (4) and (9) with (21), we can say that if $a_{0} \leqslant$ 0 then the predictor is unstable. If $a_{0}>0$, the predictor might be stable.

When we arbitrarily require $a_{0}=0$ in the (LP) method, by not estimating any bias, we force $P(1,1)=1$ whenever $B$ is nonzero, and thus force the model always to be marginally unstable. This is consistent with the fact that when we add a constant (a bias) to the impulse response of an all-pole autoregressive model, then the resulting biased sequence has a rational $z$-transform whose prediction coefficients of the denominator polynomial sum up exactly to one. This is because the added constant has a $z$-transform with a pole on the unit surface.

The occurrence of an unstable model, to which Table II refers, is judged only by the criterion $a_{0} \leqslant 0$. However, for the (LP) method, the few times when the sum was less than 1 could be attributed to roundoff errors, because it has been noticed experimentally that the (LP) method almost always results in coefficients whose sum, $P(1,1)$, is very close to unity. This last observation indicates that there is indeed a bias inherent in the image data.

\section{2-D Linear Prediction of Density Images}

Linear prediction of a signal can be viewed as a linear operator acting upon the signal. Since linear operators obey the principle of additive superposition, linear prediction is especially well suited to analysis of signals which possess additive structure. Therefore, if linear prediction is to be applied to images, the question arises, can images be modeled properly by a linear system? 
TABLE II

Average Number of Unstable Models (Percent) Obtalned by Different Approaches to Linear Prediction of INTENSITY IMAGES

\begin{tabular}{cccr}
\hline $\begin{array}{c}\text { Method/Analy sis Conditions } \\
(P, M)\end{array}$ & 3,16 & 3,32 & 8,32 \\
\hline TBLP & 22.3 & 17.2 & 4.7 \\
LMLP & 19.5 & 17.2 & 4.7 \\
LP & 42.5 & 37.5 & 28.1 \\
\hline
\end{tabular}

Images are formed by light energy from an illumination source being reflected by physical objects. Thus, Stockham [13] was led to model an image signal as a product of two basic parts. For discrete intensity image arrays,

$$
x_{I}(m, n)=i_{I}(m, n) \cdot r_{I}(m, n)
$$

where $x_{I}(m, n)$ is the discrete intensity image array, $i_{I}(m, n)$ is the illumination, and $r_{I}(m, n)$ is the reflectance component. The subscript " $I$ " refers to intensity signals. Both the intensity and the illumination signals are spatial patterns of light energy that must be positive and nonzero. The reflectance is additionally constrained to be less than unity [13]. These two basic components have distinctly different characteristics and convey different kinds of information. The illumination component models the lighting of the scene and it varies slowly across the scene, except in the case of shadows. The reflectance depends upon the nature of the objects in the scene and thus it may vary more rapidly across the scene.

Stockham [13], [14] has shown that signals modeled as a product of two components can be processed using a homomorphic system for multiplication wherein a logarithmic transformation is used to convert the multiplicative superposition into an additive superposition of signals, to which a linear prediction system may be more compatible. In the context of image signals, the logarithm of an intensity sample is termed a "density sample" [13]. Taking the logarithm of image intensities does not cause any mathematical difficulties, because the intensities are always positive. Thus, the discrete density image array is

$$
\begin{aligned}
x_{D}(m, n) & =\log \left[x_{I}(m, n)\right] \\
& =\log \left[i_{I}(m, n)\right]+\log \left[r_{I}(m, n)\right] \\
& =i_{D}(m, n)+r_{D}(m, n)
\end{aligned}
$$

where $i_{D}(m, n)$ and $r_{D}(m, n)$ represent the illumination density and the reflectance density signals, respectively. The subscript " $D$ " refers to density signals. The base of the logarithm does not play any role since a change of this base would just multiply both sides of (23) by a scaling factor. Equation (23) reveals the additive structure of the density image signal. Moreover, assuming that the illumination density, which is a slowly varying spatial pattern, does not vary appreciably over each small image region or analysis frame, we can write

$$
x_{D}(m, n)=i_{D}+r_{D}(m, n)
$$

where $i_{D}$ represents the more or less constant illumination density over each small analysis frame. Comparing (3) and (24) we can relate the illumination density to the average bias over each frame and the reflectance density to the unbiased density signal. The additive structure of the density image suggests that linear prediction may be better matched to density signals than to intensity signals.

The experimental results of applying linear prediction to density images supports this notion. For an 8 bit/pixel intensity image the intensity samples assume values from the finite range 1 to 256 . Thus, the values of the corresponding density samples will range from $\log (1)=0$ to $\log (256)=5.545$, if the natural logarithm is considered. Linear predictive analysis was applied to such density samples in exactly the same way as was done in the case of intensity images.

The resulting total normalized prediction error over the whole "Girl" image is shown in Table III, whereas Fig. 6 shows the average normalized prediction error per frame. In both cases the normalization was done by dividing by the energy of the density image signal. Table III compares the TBLP, LMLP, and LP cases for the covariance method. Fig. 6 compares the covariance versus autocorrelation method for various frame sizes for a fixed predictor order in the TBLP case. The variation of the prediction error versus predictor order for a fixed frame size was found to be small, as in the intensity case (see Fig. 5).

The results in Table III and Fig. 6 indicate that the TBLP method for bias removal is slightly to be preferred, and that the covariance formulation is superior to the autocorrelation formulation. Comparing the above results with the comparable results in the intensity case (see Table I and Fig. 4) we can conclude that linear prediction on the density image always gives a smaller normalized prediction error than on the intensity image. For the covariance method, the normalized prediction error is approximately seven to eight times smaller.

\section{E. 2-D Separable Linear Predictor}

As mentioned above, stability is a very important issue in linear prediction, and neither the covariance nor the autocorrelation method can guarantee the stability of the resulting inverse prediction error filter in two-dimensional linear predictive analysis [11]. Only in the one-dimensional case can the autocorrelation method guarantee stability [5]. Thus, if the prediction error filter is structured as the product of two 1-D prediction error filters-each one predicting along a different direction in the $(m, n)$ plane-then, by using the 1-D autocorrelation method, the parameters of each individual filter can be found so that stability is guaranteed. For example, suppose that we desire a $Q \times Q 2-D$ separable prediction mask. Its corresponding prediction error filter and prediction error sequence will be respectively

$$
\begin{aligned}
A\left(z_{1}, z_{2}\right)= & \left(1-\sum_{k=1}^{Q-1} a(k) z_{1}^{-k}\right)\left(1-\sum_{l=1}^{Q-1} b(l) z_{2}^{-l}\right) \\
c(m, n)= & x(m, n)-\sum_{k=1}^{Q-1} a(k) x(m-k, n) \\
& -\sum_{l=1}^{Q-1} b(l) x(m, n-l) \\
& +\sum_{k=1}^{Q-1} \sum_{l=1}^{Q-1} a(k) b(l) x(m-k, n-l) .
\end{aligned}
$$


TABLE III

Total Normalized Prediction Error (Percent) for linear Prediction of Density Images

\begin{tabular}{cccc}
\hline $\begin{array}{c}\text { Method/Analysis Conditions } \\
(P, M)\end{array}$ & 3,32 & 3,16 & 8,32 \\
\hline TBLP & 0.0993 & 0.0885 & 0.0841 \\
LMLP & 0.0998 & 0.0903 & 0.0843 \\
LP & 0.1008 & 0.0932 & 0.0860 \\
\hline
\end{tabular}

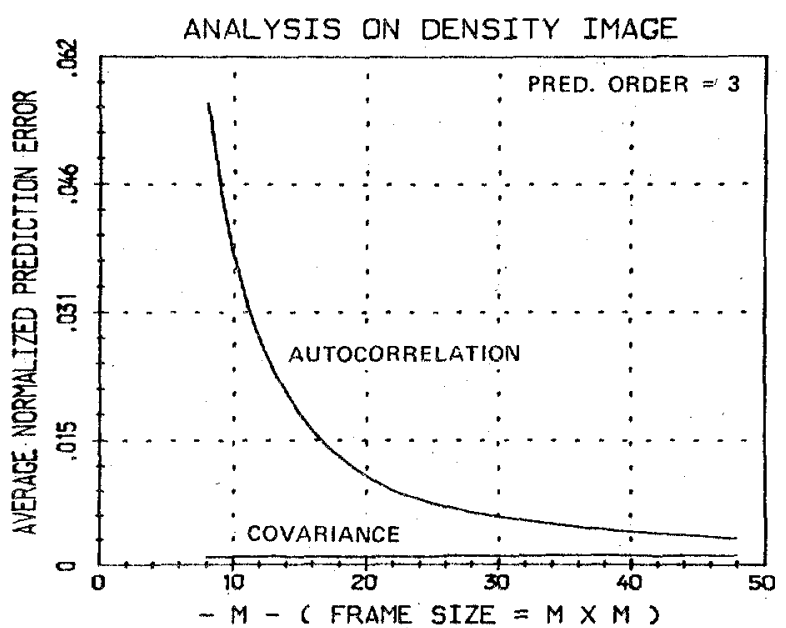

Fig. 6. Variation of prediction error versus frame size for density images $(P=3)$.

In general, to find the coefficients of a separable predictor, $\{a(k), b(k), k=1, \cdots, Q-1\}$, we must minimize the square norm of $e(m, n)$ by taking partial derivatives with respect to the coefficients, setting equations equal to zero, and solving the resulting system. However, this will be a formidable task since it is evident from (26) that the resulting system of equations is nonlinear. A suboptimum solution is obtained when the 2-D separable filter is realized as a cascade of two filters with the error sequence being minimized at the output of each filter separately. In other words, prediction is done first along one direction and then along the other. If $s(m, n)$ is the output of the first prediction error filter

$$
s(m, n)=x(m, n)-\sum_{k=1}^{Q-1} a(k) x(m-k, n)
$$

then the optimal $\{a(k)\}$ which minimizes

$$
E_{1}=\sum_{m} \sum_{n} s^{2}(m, n)
$$

can be found by using the autocorrelation method on an $M \times$ $M$ frame of the signal $x(m, n)$. The well-known Levinson recursion [5] can be used to find the $a(k)$ 's, and the resulting 1-D prediction error filter will be stable. Similarly the output of the second 1-D prediction error filter is given by

$$
e(m, n)=s(m, n)-\sum_{l=1}^{Q-1} b(l) s(m, n-l)
$$

where $s(m, n)$ is given by (27). The optimal coefficients $\{b(l)\}$ which minimize the error

$$
E_{2}=\sum_{m} \sum_{n} e^{2}(m, n)
$$

can be found by using the autocorrelation method on a $M \times M$ frame of the signal $s(m, n)$. Again, 1-D Levinson recursion can be employed to give a stable filter.

At this point, we should emphasize that the procedure is suboptimum; i.e., $E_{2}>E$, where $E$ given by (8) represents the error of the general 2-D predictor and $E_{2}$ represents the error of the suboptimum 2-D separable predictor. Throughout all the above discussion we omitted the problem of estimating the bias, because for the autocorrelation method [see (19) and (20)] this problem is decoupled from the problem of estimating the optimal prediction coefficients.

Example: For the $2 \times 2$ separable predictor there are only two unknown prediction coefficients: $\{a, b\}$. The 2-D prediction error filter and the corresponding error sequence will be

$$
\begin{aligned}
A\left(z_{1}, z_{2}\right) & =\left(1-a z_{1}^{-1}\right)\left(1-b z_{2}^{-1}\right) \\
e(m, n) & =s(m, n)-b s(m, n-1)
\end{aligned}
$$

where

$$
s(m, n)=x(m, n)-a x(m-1, n) .
$$

The optimal coefficients $(a, b)$ will be

$$
a=\frac{R_{x}(1,0)}{R_{x}(0,0)}, \quad b=\frac{R_{s}(0,1)}{R_{s}(0,0)}
$$

where the 2-D autocorrelation lags $R_{x}(\cdot, \cdot)$ are given by (18), from which $R_{s}(\cdot, \cdot)$ is also given if we replace the signal $x(m, n)$ by $s(m, n)$. Using the Cauchy-Schwarz inequality, it is easy to prove that $|a|<1$ and $|b|<1$. Therefore, the separable prediction error filter of (31) has a stable inverse.

Fig. 7 shows the variation of the average normalized prediction error versus different frame sizes, using a 2-D separable linear predictor with the autocorrelation method for a fixed predictor order $P=3$. By comparing Figs. 4 and 7 , we see that, in the autocorrelation method, both the separable and the nonseparable predictor have the same performance, except for a small increase (3-4 percent) of the prediction error in the separable case. However, the separable predictor still gives a two to four times greater prediction error than the predictor in the covariance method.

\section{Adaptive Predictive Image Coding}

So far, we have discussed several theoretical issues concerning the modeling, performance, and stability of 2-D linear prediction applied to monochromatic image signals. Onedimensional linear prediction constitutes the framework for the very effective predictive coding of speech at low bit rates [15]. Following a similar approach, we applied 2-D linear prediction to predictive coding of still monochromatic images in an intraframe ADPCM with both adaptive prediction and adaptive quantization.

\section{A. Adaptive Prediction}

The system studied is illustrated in Fig. 8. The heart of this system is a basic DPCM system. Its predictor forms an estimate, $\widetilde{x}(m, n)$, of an image sample to be coded, $x(m, n)$, from past reconstructed samples at the receiver, $\hat{x}(m, n)$. The difference between $x(m, n)$ and its estimate $\tilde{x}(m, n)$ is the difference signal $d(m, n)$, which is quantized and encoded for transmission. In adaptive systems, the predictor is adapted to 


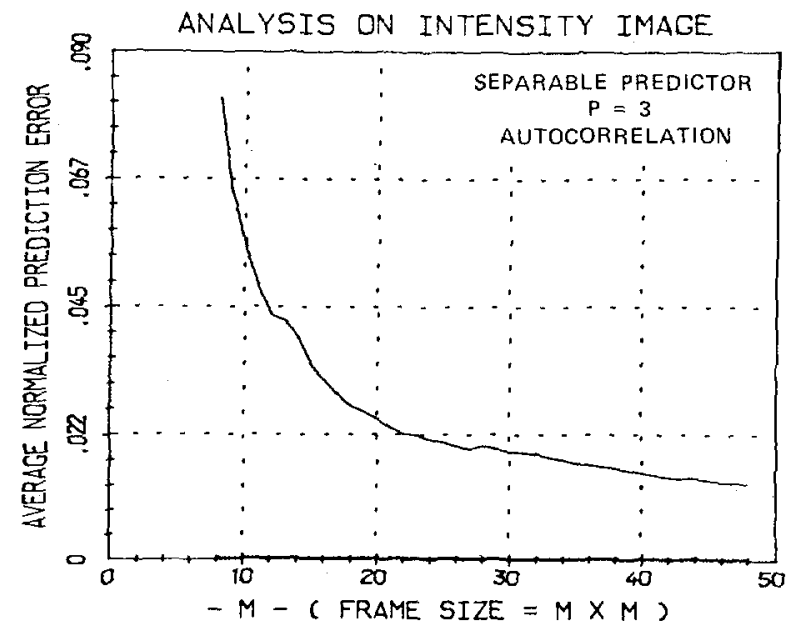

Fig. 7. Variation of prediction error versus frame size for a 2-D separable predictor on intensity images $(P=3)$.

(a)

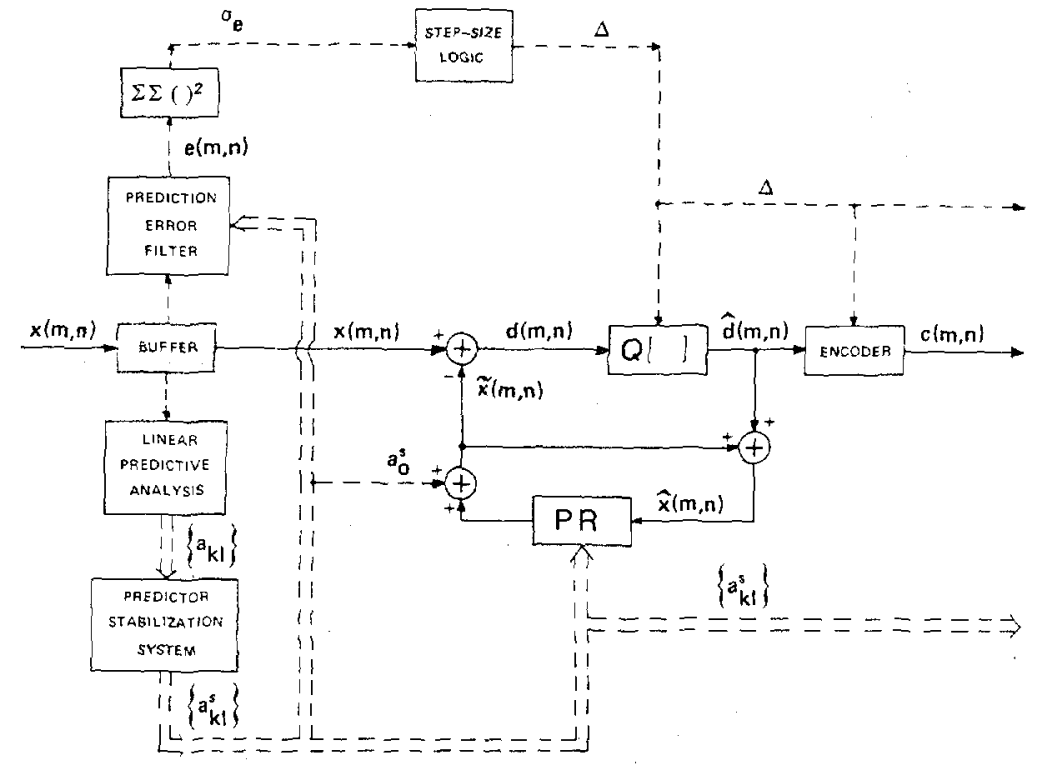

(b)

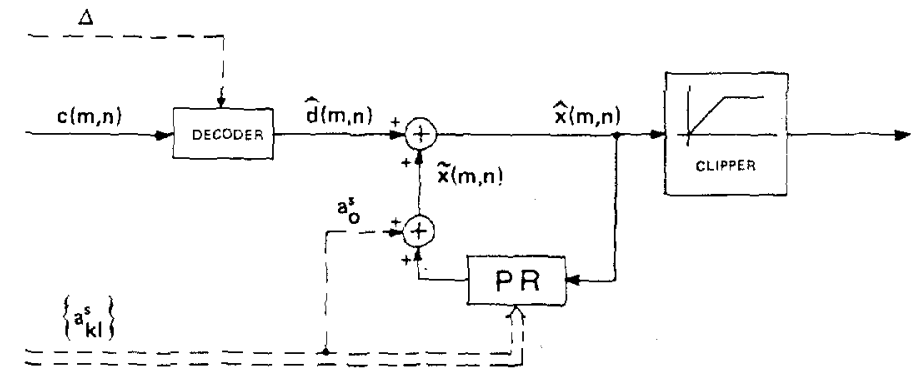

Fig. 8. (a) Adaptive DPCM coder for images. (b) Decoder for ADPCM.

the nonstationarity of the image by using 2-D linear predictive analysis to obtain estimates of the LPC coefficients in (1) over small image regions. It should be noted that, although the optimal coefficients are obtained from the unquantized samples $x(m, n)$, the predictor operates on the reconstructed samples $\hat{x}(m, n)$ (see Fig. 8). However, this results in only a small loss in optimality when the quantization error is small; i.e, $x(m, n) \approx \hat{x}(m, n)$.

Stabilizing Technique: At the receiver of Fig. 8 , the quan- tized difference signal $\hat{d}(m, n)$ excites the inverse prediction error filter and generates the reconstructed image signal $\hat{x}(m, n)$. Therefore, the stability of this filter must be somehow guaranteed. This can be achieved by using a 2-D separable linear predictor, as discussed earlier, but this sacrifices the full generality of 2-D linear prediction. Thus, another approach which we have followed with good success is to use a suboptimal stable model obtained by multiplying each predic. tion coefficient by a constant $\beta$ such that $\beta \cdot P(1,1)<1$, in 
cases where $P(1,1) \geqslant 1$ and the model was necessarily unstable according to (21). The value chosen for $\beta$ was approximately $\approx 0.99 / P(1,1)$, and it varied for each frame of the image. When the stabilizing constant is used, the bias coefficient $a_{0}$ must be corrected so that (4) still holds for $B$ as originally estimated

$$
a_{0}^{s}=B[1-\beta P(1,1)] .
$$

We have always found the resulting model to be stable even though (21) is only a necessary condition. Indeed, since stabilizing the model results in higher prediction error in the coder, we have found it best to place a lower limit on $\beta\left(\beta_{\min } \approx\right.$ 0.75 ), and accept an unstable model for very few regions of the image.

With this modification, the difference equations which describe the operation of the coder and decoder in Fig. 8 become

$$
d(m, n)=x(m, n)-\beta \cdot \sum_{\substack{k \\(k, l) \in \Pi}} a(k, l) \hat{x}(m-k, n-l)-a_{0}^{s}
$$

$$
\hat{x}(m, n)=\beta \cdot \sum_{\substack{k \\(k, l) \in \Pi}} a(k, l) \hat{x}(m-k, n-l)+a_{0}^{s}+\hat{d}(m, n) \text {. }
$$

\section{B. Adaptive Quantization}

In order to achieve bit rates below $1 \mathrm{bit} / \mathrm{pixel}$, the difference signal $d(m, n)$ was quantized with a three-level center-clipping quantizer. Similar quantizers have been used in speech and image quantization as reported in [16] and [17], respectively. The input-output characteristic of this three-level quantizer is illustrated in Fig. 9, and it is given by the equation

$$
d(m, n)=\left\{\begin{array}{cl}
\Delta, & d(m, n) \geqslant \theta \\
0, & -\theta<d(m, n)<\theta \\
-\Delta, & d(m, n) \leqslant-\theta .
\end{array}\right.
$$

The threshold $\theta$ determines the percentage of the dynamic range of the input signal to the quantizer to be assigned a zero value. The behavior of the quantizer can be varied by varying $\theta$ proportionally to the step-size $\Delta$. For instance, if $\theta=0$, the quantizer has only two levels, while $\theta=\Delta / 2$ corresponds to a uniform three-level quantizer. Finally, for $\theta>\Delta / 2$ we have a three-level center-clipping quantizer. In order to achieve a bit rate for the difference signal of at most $1 \mathrm{bit} / \mathrm{pixel}$ using codewords of fixed length, it is necessary to use a two-level quantizer. However, the dynamic range of the difference signal is often too large to be handled properly by a two-level quantizer. Such a coarse quantization is a major source of visible distortions in the reconstructed image. With only two levels it is difficult to avoid both peak-clipping of the difference signal and granular distortion. Peak-clipping results in smeared edges in the reconstructed image, while a large step-size chosen to avoid peak-clipping introduces large amounts of granular noise. In contrast, a three-level quantizer offers the alternative of having the zero middle level for small amplitudes of the difference signal plus two side-levels for handling the large ampli-

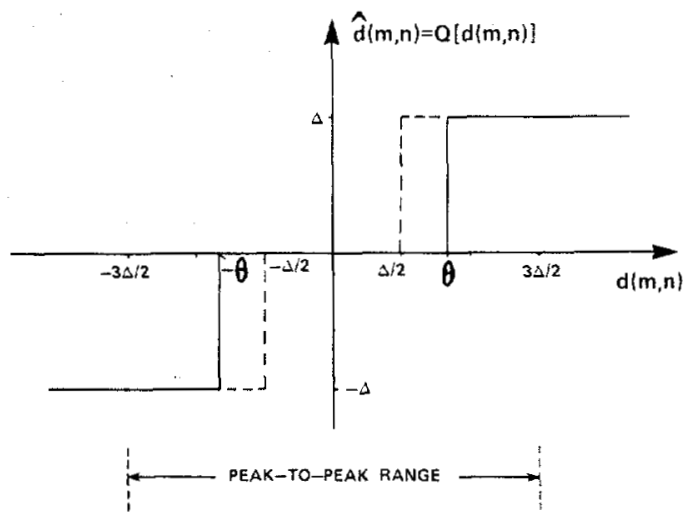

Fig. 9. (-): Input-output characteristic of a three-level centerclipping quantizer. $(--)$ ): Three-level uniform quantizer.

tude portion of the difference signal. The first-order entropy of the output signal from a two-level quantizer in ADPCM is very nearly equal to $1 \mathrm{bit} /$ pixel. However, by increasing the threshold $\theta$ of the three-level quantizer, a large number of zero values is produced at the output, and the entropy of the quantized difference signal can be made significantly smaller than $1 \mathrm{bit} / \mathrm{pixel}$. This can be exploited by employing block-coding techniques and encoding efficiently at average bit rates below $1 \mathrm{bit} /$ pixel.

The 2-D quantization error sequence $q(m, n)$ is defined as the difference between the input and output signals to the quantizer. Due to the feedback loop around the quantizer of Fig. 8 , the reconstruction error between $x(m, n)$ and $\hat{x}(m, n)$ is equal to the quantization error $q(m, n)$. Therefore, for high fidelity image transmission, $q(m, n)$ must be as small as possible. This can be done by first adapting the parameters of the quantizer over each $M \times M$ image frame, and second, by designing an "optimum" quantizer which takes into consideration the amplitude distribution of the difference signal. Neglecting the stabilizing factor $\beta$ and manipulating the equations of the ADPCM loop, we can write

$$
d(m, n)=e(m, n)-\sum_{\substack{k \\(k, l) \in \Pi}} \sum_{l} a(k, l) q(m-k, n-l) .
$$

Thus, we see that the difference signal is equal to the prediction error signal $e(m, n)$ plus a filtered version of the quantization error signal $q(m, n)$. This fact makes it impossible to relate the parameters of the quantizer (step-size and threshold) directly to the variation of $d(m, n)$, since $q(m, n)$ is unknown in advance. One possible solution is to relate them to the variation of the prediction error signal $e(m, n)$, which can be obtained in advance. The motivation for this was the experimental observation that both $d(m, n)$ and $e(m, n)$ possess a Laplacian amplitude distribution. Thus, the adaptation procedure for each $M \times M$ image frame is defined as

$$
\begin{aligned}
\Delta & =D \cdot \sigma_{e} \\
\theta & =K \cdot \sigma_{e}
\end{aligned}
$$

where $\sigma_{e}$ denotes the standard deviation of the zero-mean prediction error signal $e(m, n)$ over the $M \times M$ image frame. The parameter $D$ controls the dynamic range of the quantizer and the tradeoff between granular noise and peak-clipping. In- 
creasing the value of parameter $K$ reduces the entropy of the quantized difference signal. For a certain fixed $K$, the optimum choice of $D$ for minimum quantization error can be guided by knowledge of the amplitude distribution of $d(m, n)$ and $e(m, n)$. An empirically determined estimate for $D$ is $D=$ 1.5 for $K=0$ (two-level quantizer) and $D=2$ for $K / D>0.5$ (three-level center-clipping quantizer) [18] .

Encoding of the Quantized Difference Signal and the Side Information: For a three-level quantizer, the quantized difference signal contains at most three amplitude levels: $-\Delta, 0$, and $\Delta$. If we consider this as a source alphabet of three letters $(-1$, $0,1)$ and segment the entire quantized difference image into 1-D or 2-D nonoverlapping and touching blocks of $L$ samples, then the $L$ th-order joint entropy in bits per sample of the image is

$$
\begin{aligned}
H_{L}= & -(1 / L) \cdot \sum_{x_{1}} \cdots \sum_{x_{L}} p\left(x_{1}, \cdots, x_{L}\right) \\
& \cdot \log _{2} p\left(x_{1}, \cdots, x_{L}\right)
\end{aligned}
$$

where $x_{i}$ represents an encoded quantized difference sample whose value is $-1,0$, or 1 and $p\left(x_{1}, \cdots, x_{L}\right)$ denotes the $L$ thorder joint probability of the $L$ samples $x_{1}, \cdots, x_{L}$ of one block. In practice we measure histograms instead of probability distributions. It is well known [19] that by using optimum Huffman encoding of the blocks we can achieve an average bit rate $R_{\text {HUF }}$ which is arbitrarily close to $H_{L}$ for large $L$ : $H_{L} \leqslant R_{\mathrm{HUF}}<H_{L}+1 / L$.

If $\theta=0$, we have only two quantization levels. Therefore, we can use codewords of fixed length to encode the quantized difference image at $1 \mathrm{bit} / \mathrm{sample}$. Alternatively, we could use Huffman codewords of variable length to encode blocks of $L$ samples. In our ADPCM coder, the first-order entropy of binary quantized difference images was found to be $\approx 1$ bit/ sample. However, their 16th-order entropy was equal to about $\approx 0.7$ bits/sample. Thus, by using Huffman coding for 2-D blocks with $4 \times 4$ samples we can encode this binary quantized difference signal at an average rate $R_{d}$ very close to 0.7 bits/sample: $0.7 \leqslant R_{d}<0.7+\left(\frac{1}{16}\right)$.

If $\theta \neq 0$, we have three quantization levels. To use in this case Huffman coding for blocks of 16 samples would be highly impractical, because the Huffman table would contain $3^{16}$ entries. Therefore, to encode the quantized difference image, we used the coding procedure described in [16], which exploits the fact that, due to the large number of zero values in the output from the quantizer, the first-order entropy in (42) is very often smaller than $1 \mathrm{bit} / \mathrm{sample}$. That is, code words of variable length were assigned to blocks having a fixed length of $L$ samples. The average number of bits required per sample is

$$
R_{d}=(1 / L) \cdot \sum_{n=0}^{L} \operatorname{Pr}(n) \cdot b(n)
$$

where $b(n)$ denotes the number of bits required for a block with $n$ zeros and $\operatorname{Pr}(n)$ is the probability that a block of $L$ samples contains $n$ zero samples. The following example will clarify the efficiency of the above procedure. An image was coded through the simulated ADPCM system of Fig. 8 with a three-level center-clipping quantizer yielding a first-order entropy of $H_{1}=0.701$ bits/sample which corresponds to a percentage of zero-valued samples equal to 86 percent. By encoding blocks of $L=4$ samples long, an average bit rate of $0.734 \mathrm{bits} / \mathrm{sample}$ resulted for the quantized difference signal. This bit-rate is only 1.05 times the entropy. By using larger blocks of $L=8$ samples the average bit rate was further reduced to only 0.709 bits/sample, which is consistent with the noiseless coding theorem for binary transmission [19] .

The above proposed block-coding schemes have the advantage that they achieve high coding efficiency, enabling trans. mission of images at rates below $1 \mathrm{bit} / \mathrm{pixel}$. Their disadvantage is that they make use of variable-length codes so that a buffer must be provided between the variable length codes and a uniform bit rate channel. Also, the variable-length codes must be designed so as to provide protection against a loss of synchronization in the presence of channel errors. Also note that a change of the source probabilities would require a new code mapping to ensure minimal average length.

In addition to the above bit rate $R_{d}$ for the quantized difference signal, we must also transmit information about the $P+1$ predictor parameters $\left\{a(k, l), a_{0}\right\}$ and the step size $\Delta$ (also referred to as "side information"). The dynamic range of the prediction coefficients $a(k, l)$ of a stable $2 \times 2$ predictor is necessarily $(-1,1)$. For $3 \times 3$ predictors, we experimentally found that the prediction coefficients were always absolutely less than 1. Motivated by the established techniques in the area of speech coding [5] for quantizing reflection coefficients, instead of directly quantizing a prediction coefficients $a(k, l)$, we quantized the quantity $\log [(1-a(k, l)) /(1+$ $a(k, l))]$ for all $(k, l) \in \Pi$ using a uniform quantizer in the implied range. We found that by using quantized coefficients with 6-10 bits/coefficient, the SNR of the resulting coded images was about $0.2 \mathrm{~dB}$ less than the resulting SNR when using unquantized coefficients. To quantize the bias coefficient $a_{0}$, we can quantize instead the bias $B$ whose dynamic range is known $(0-255$ for 8 bit/pixel intensity images) and then obtain $a_{0}$ using (4). The dynamic range for the step size was experimentally set equal to half the range for the bias $B$. Both the bias and the step size were quantized using logarithmic uniform quantizers. A typical bit allocation used in our ADPCM scheme was: 6 bits for each prediction coefficient, 7 bits for the bias, and 6 bits for the step size on every analysis frame. If $n_{s}$ denotes the average number of bits per sideinformation parameter, then the total average bit rate of the coded image is

$$
R=R_{d}+(P+2) \cdot n_{s} / M^{2} .
$$

For the above bit allocation, the side information for $32 \times 32$ frames requires 0.03 and 0.06 bits/pixel for predictor orders of $P=3$ and $P=8$, respectively. For $16 \times 16$ frames, the above rates for the side information increase to 0.12 and 0.24 bits/pixel.

\section{Experimental Results}

The ADPCM scheme of Fig. 8 with both adaptive prediction and adaptive quantization was applied in coding monochromatic still images. For simplicity, all the following results will 


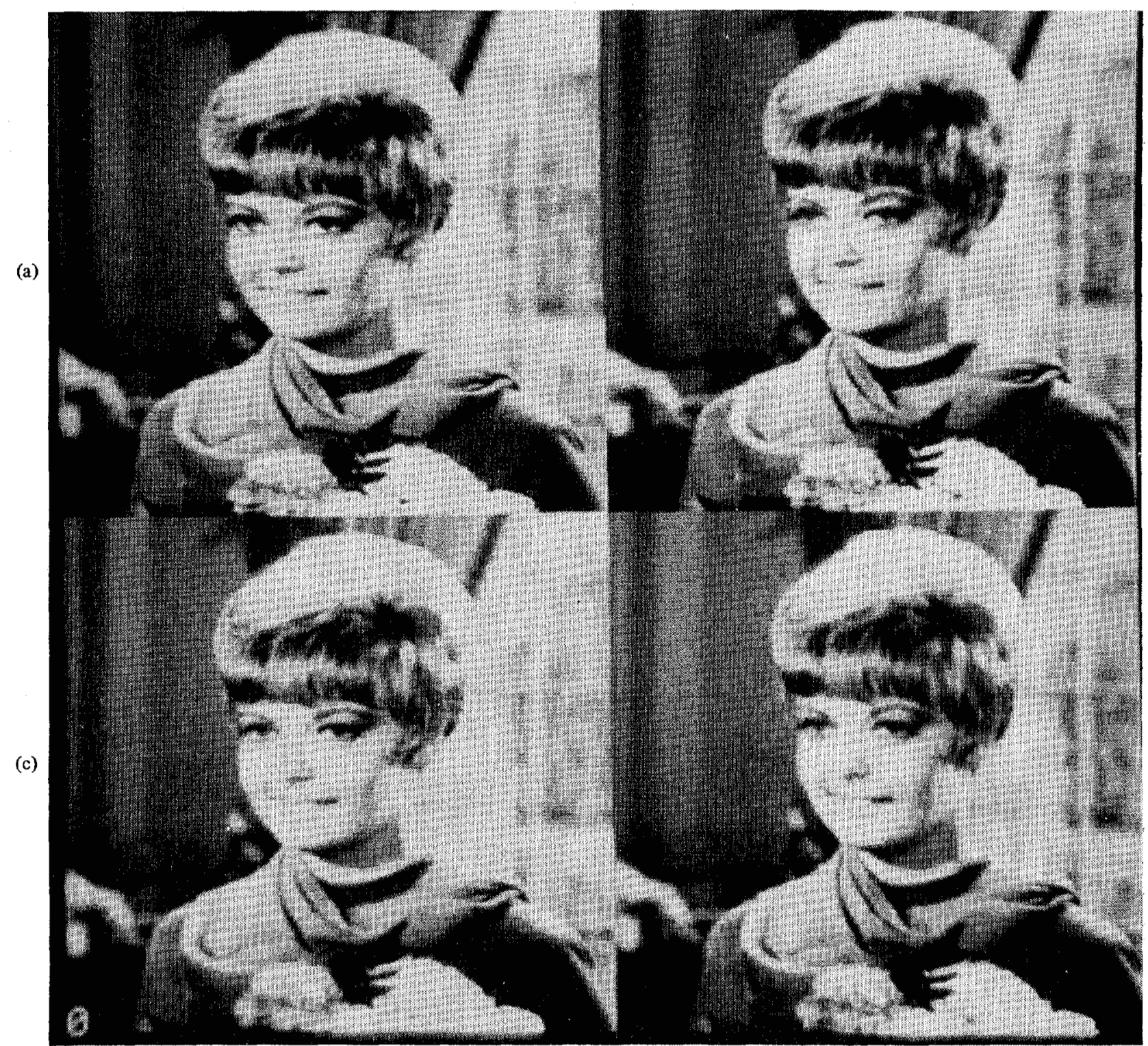

(b)

Fig. 10. Coded intensity images using a two-level quantizer $(D=1.5)$. (a) $P=8, M=16, R=0.96 \mathrm{bit} / \mathrm{pel}$. (b) $P=8, M=32, R=0.78 \mathrm{bit} /$ pel. (c) $P=3, M=16, R=0.84 \mathrm{bit} / \mathrm{pel}$. (d) $P=3, M=32, R=0.75$ bit/pel.

refer to the head-and-shoulders image of Fig. 2(a), but similar results were obtained for other images as well. The adaptation took place by dividing the whole image in $M \times M$ frames where $M$ was equal to 16 or 32 . The coefficients of the 2-D linear predictor for each frame were obtained by using the covariance method, with the bias being estimated together with the predictor coefficients (TBLP method). The adaptive quantizer was used with either two or three quantization levels.

For measuring the fidelity of the reconstructed images, we employed a widely used [7] version of signal-to-noise ratio defined as

$$
\text { SNR }=10 \log _{10} \frac{(\text { Peak-to-peak value of original image data })^{2}}{\frac{1}{N^{2}} \sum_{m=1}^{N} \sum_{n=1}^{N}[x(m, n)-\hat{x}(m, n)]^{2}}
$$

where $N^{2}$ represents the number of pixels of the original image. At the receiver of Fig. 8, a clipper was used after the reconstruction procedure. The clipper resets the values of the reconstructed image samples $\hat{x}(m, n)$ to within the limits of the dynamic range of an 8 bit imaging system (i.e., 0-255). The need for the clipper arises because the nonlinearities of the quantizer and the numerical instabilities of the predictor always create a small percentage of samples $(\approx 1$ percent $)$ exceeding the specified dynamic range. Alternatively, inserting a clipper before the predictor both at the transmitter and at the receiver results in a slightly lower SNR and a greater percentage of clipped levels.

Coding of Intensity Images: By applying adaptive predictive coding to the intensity image signal, reconstructed images of full intelligibility and good fidelity resulted. Fig. 10 shows a set of four reconstructed images which were coded by using a two-level quantizer and different frame sizes as well as differ- 
TABLE IV

SNR FOR CODED INTENSITY IMAGES USING a Two-Level Quantizer (See Fig. 10)

\begin{tabular}{ccc}
\hline $\begin{array}{c}\text { Predictor } \\
\text { Order }\end{array}$ & $\begin{array}{c}\text { Frame } \\
\text { Size }\end{array}$ & $\begin{array}{c}\text { SNR } \\
(\mathrm{dB})\end{array}$ \\
\hline 3 & $32 \times 32$ & 30.6 \\
8 & $32 \times 32$ & 31.1 \\
3 & $16 \times 16$ & 31.2 \\
8 & $16 \times 16$ & 32.2 \\
\hline
\end{tabular}

(a)

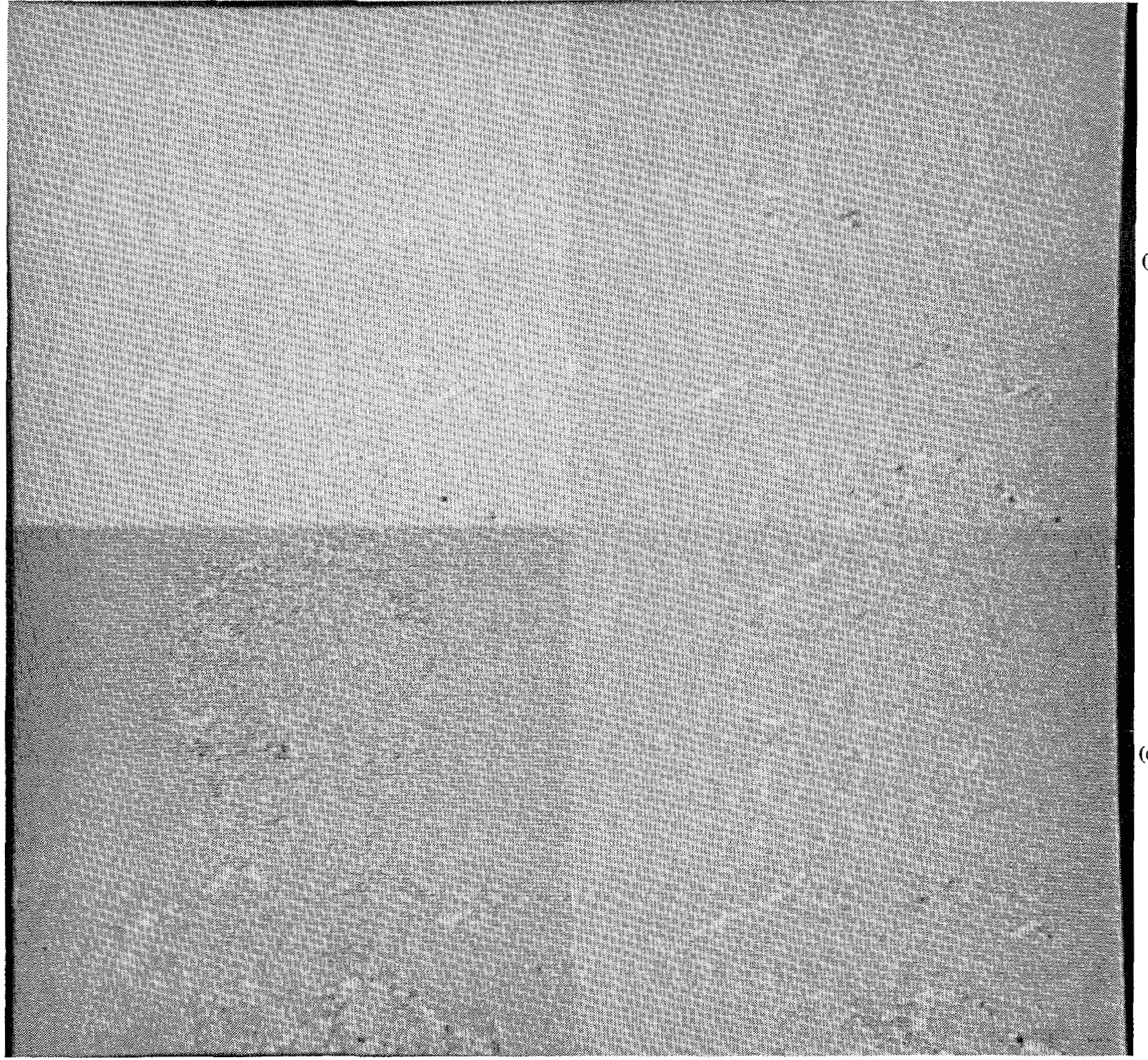

Fig. 11. Quantization error images for the respective coded images of Fig. 10. (a) $P=8, M=16$. (b) $P=8, M=32$. (c) $P=3, M=16$. (d) $P=3, M=32$.

ent predictor orders. Table IV contains the resulting SNR's. The corresponding quantization error images are shown in Fig. 11. They were formed by mapping the difference between original and reconstructed images onto the original range from 0-255 for display. Table IV indicates that using both smaller frames and higher predictor orders gives a higher SNR. However, the $16 \times 16$ frames require a bit rate for the side information which is four times higher than for $32 \times 32$ frames. Subjective image quality tests indicate that the $3 \times 3$ predictor gives images with sharper edges than a $2 \times 2$ predictor, without significantly increasing the additional required bit rate.

Fig. 12 illustrates a set of four reconstructed images which were obtained by fixing the predictor order at $P=3$ and the 


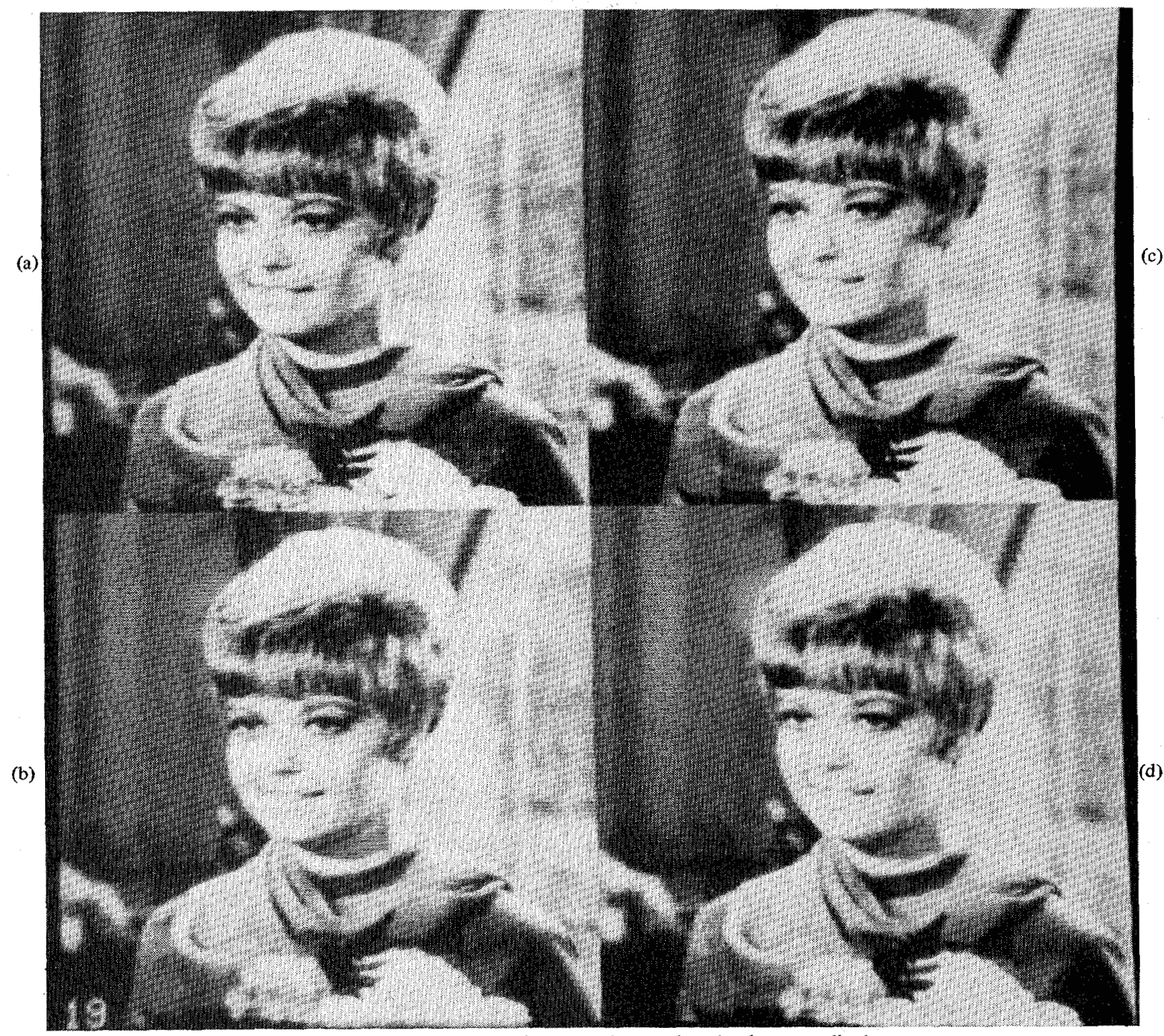

Fig. 12. Coded intensity images using a three-level center-clipping quantizer ( $D=2, P=3, M=32$ ). (a) $R=1.03 \mathrm{bit} / \mathrm{pel}$. (b) $R=0.93$ bit $/$ pel. (c) $R=0.83 \mathrm{bit} / \mathrm{pel}$. (d) $R=0.74 \mathrm{bit} / \mathrm{pel}$.

TABLE V

SNR of CODED INTENSITY IMAGES VERSUS ENTROPY of Difference Signal for Three-Level. Quantizer with $D=2, M=32, P=3$ (See Fig. 12)

\begin{tabular}{ccc}
\hline \hline$K$ & $\begin{array}{c}\text { First-Order Entropy } \\
\text { (bits/pixel) }\end{array}$ & SNR (dB) \\
\hline 2.0 & 0.707 & 30.3 \\
1.7 & 0.804 & 31.6 \\
1.5 & 0.900 & 32.6 \\
1.3 & 1.002 & 33.4 \\
\hline
\end{tabular}

frame size at $M=32$, by using a three-level center-clipping quantizer, and by varying its threshold so that entropies of approximately $0.7,0.8,0.9$, and $1 \mathrm{bit} /$ pixel result. The major observation of Fig. 12 is that contouring effects become obvious as the entropy is reduced. Consistent with this observation is the fact that the SNR becomes smaller at lower entropies, as shown in Table V. Comparing Figs. 10 and 12 leads to the following conclusions. a) For the same average bit rate of $\approx 0.7 \mathrm{bits} /$ pixel, predictor order, and frame size, both the three-level and the two-level quantizers result in the same SNR. Moreover, in the case of three quantization levels, the reconstructed image appears to have sharper edges, less granular noise, but some contouring effects. b) For the same bit rate of $1 \mathrm{bit} /$ pixel using codewords of variable length for the three-level and of fixed length for the two-level quantizer, the three-level quantizer gives images with almost no contouring effects and some aspects of superior fidelity, such as sharper edges, less granular noise, and higher SNR (approximately $3 \mathrm{~dB}$ more).

Coding of Density Images: Recall that linear prediction performed better on density images than on intensity images. It is therefore of interest to apply ADPCM coding to density images. This procedure is summarized in Fig. 13, where the image density signal $x_{D}(m, n)$ is coded by an ADPCM configuration identical to the one in Fig. 8 . The reconstructed den- 


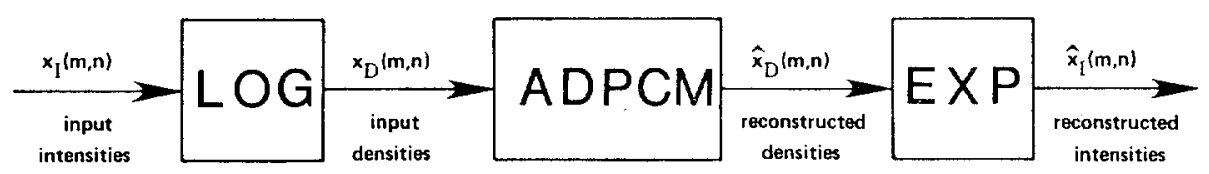

Fig. 13. ADPCM on the density representation of images.

sity image $\hat{x}_{D}(m, n)$ yields after exponentiation the reconstructed intensity image $\hat{x}_{I}(m, n)$. There exist two different reconstruction errors: the one between the original and the reconstructed intensity image which is expressed by the "intensity SNR" in (45), and the error between original and reconstructed density image which can be expressed by the "density SNR," given by (45) if we substitute density values. The density SNR measures the performance of ADPCM on the density image. The intensity SNR measures the performance of the overall coding scheme of Fig. 13.

As far as the density SNR is concerned, the experimental results were in agreement with the very good performance of linear prediction on the density image. Namely, for $P=8$ and $M=32$, the resulting density SNR was about $32.5 \mathrm{~dB}$. However, of greater importance is the visible SNR which is the intensity SNR, which was about $28.5 \mathrm{~dB}$. The reconstructed intensity image is shown in Fig. 14, where some "black spots" disturb the uniformity of the image and make it appear that the image is being seen through a "dirty window." The difference in SNR upon moving from densities to intensities is not yet well understood. A reason might be that the quantization errors are magnified nonlinearly by the exponentiation. One advantage of coding the density image is that the reconstructed intensity image is guaranteed to be positive, as a true image signal should be, because of the exponentiation of the reconstructed density image.

Coding of the Perceptual Visual Domain: Stockham [13], motivated by a model for the early portions of the human visual system depicted in Fig. 15, suggests that image processing be done after the image has been transformed by the visual model. This model assumes that the eye is logarithmically sensitive. Moreover, the densities are linearly processed by a highpass spatial linear filter $V(F)$. Stockham's empirical best estimate for $V(F)$ was

$$
V(F)=742 /\left(661+F^{2}\right)-2.463 /\left(2.459+F^{2}\right)
$$

where $F$ is the radial spatial frequency in cycles per degree, and the 2-D frequency response of the eye is assumed to be circularly symmetric.

Motivated by Stockham's argument we applied ADPCM to the representation of the image that results from processing the image with this visual model, the "perceptual visual domain." As summarized in Fig. 16, the above procedure consists of transforming the intensities to densities, filtering the densities by the high-pass spatial filter $V(F)$, coding the filtered densities by using the scheme of Fig. 8 , inverse filtering the reconstructed densities by the inverse low-pass spatial filter $V^{-1}(F)$, and finally exponentiating to end up with reconstructed intensities. To implement digitally a sampled version of $V(F)$, we used sampling frequencies at 20 or 40 samples/ ${ }^{\circ}$ corresponding to cutoff frequencies for $V(F)$ of about $F_{c 0}=$

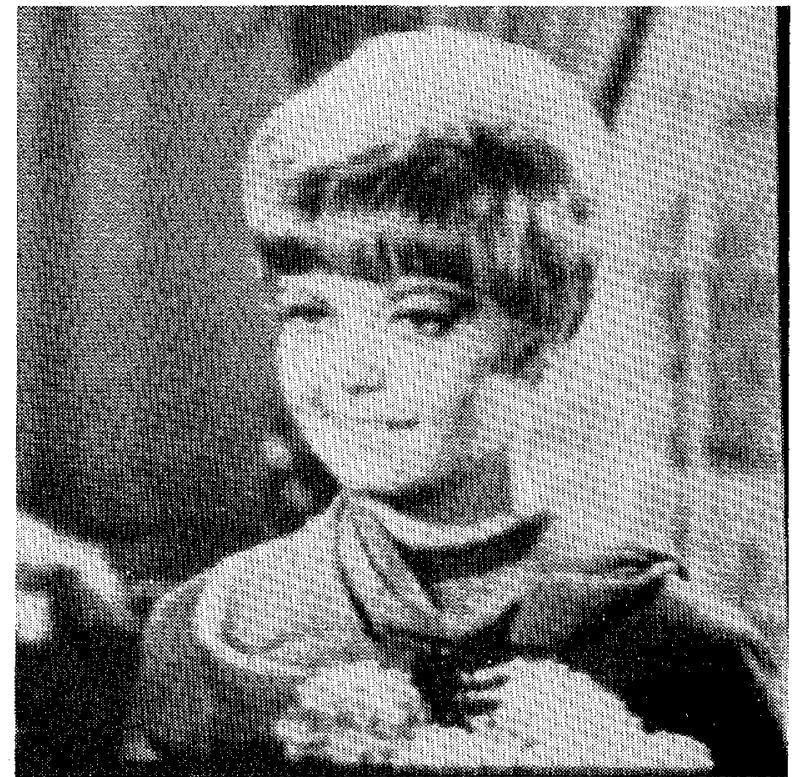

Fig. 14. Reconstructed image from ADPCM of the density representation $(K=0, D=1.5, P=8, M=32, R=0.78 \mathrm{bit} / \mathrm{pel}, \mathrm{SNR}=28.5 \mathrm{~dB})$.

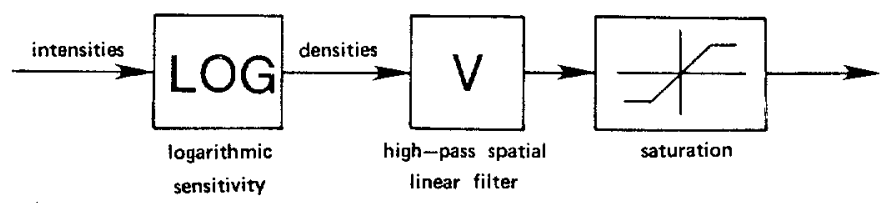

Fig. 15. An approximate model for the processing characteristics of early portions of the human system (after [13]).

10 or 20 samples $/^{\circ}$, respectively. These choices resulted from the assumption that a convenient viewing angle for a $256 \mathrm{X}$ 256 image is $6^{\circ}$, yielding a spatial sampling frequency of about 40 samples $/^{\circ}$.

The reconstructed images from coding the perceptual visual domain are shown in Fig. 17, where a two-level quantizer and various predictor orders and cutoff frequencies for $V(F)$ were used. The corresponding intensity SNR was about 26.7$28.2 \mathrm{~dB}$. Hence, from the viewpoint of SNR, coding in the perceptual visual domain instead of the density domain does not offer any advantage. From the viewpoint of fidelity, however, the black spots of the density coded image (see also Fig. 14) disappeared, verifying indeed Stockham's hypothesis that the visual-model transformation of an image increases tolerance to quantization distortions. Indeed, Fig. 18 compared with Fig. 12(d) shows that the contouring effects, which appeared in the intensity coded images by using a three-level center-clipping quantizer at bit rates below $1 \mathrm{bit} / \mathrm{pixel}$, are not evident if the output of the visual model is coded. However, the reconstructed image of Fig. 18 has another kind of coding distortion: there are some small regions of the image where the 


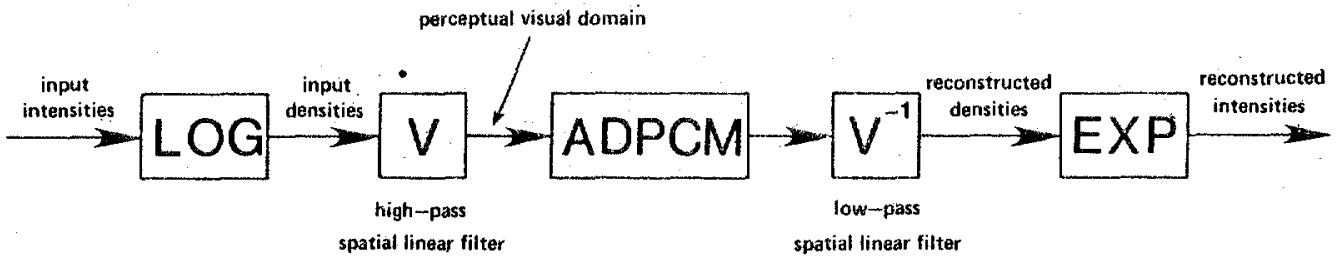

Fig. 16. ADPCM on the perceptual visual representation of images.

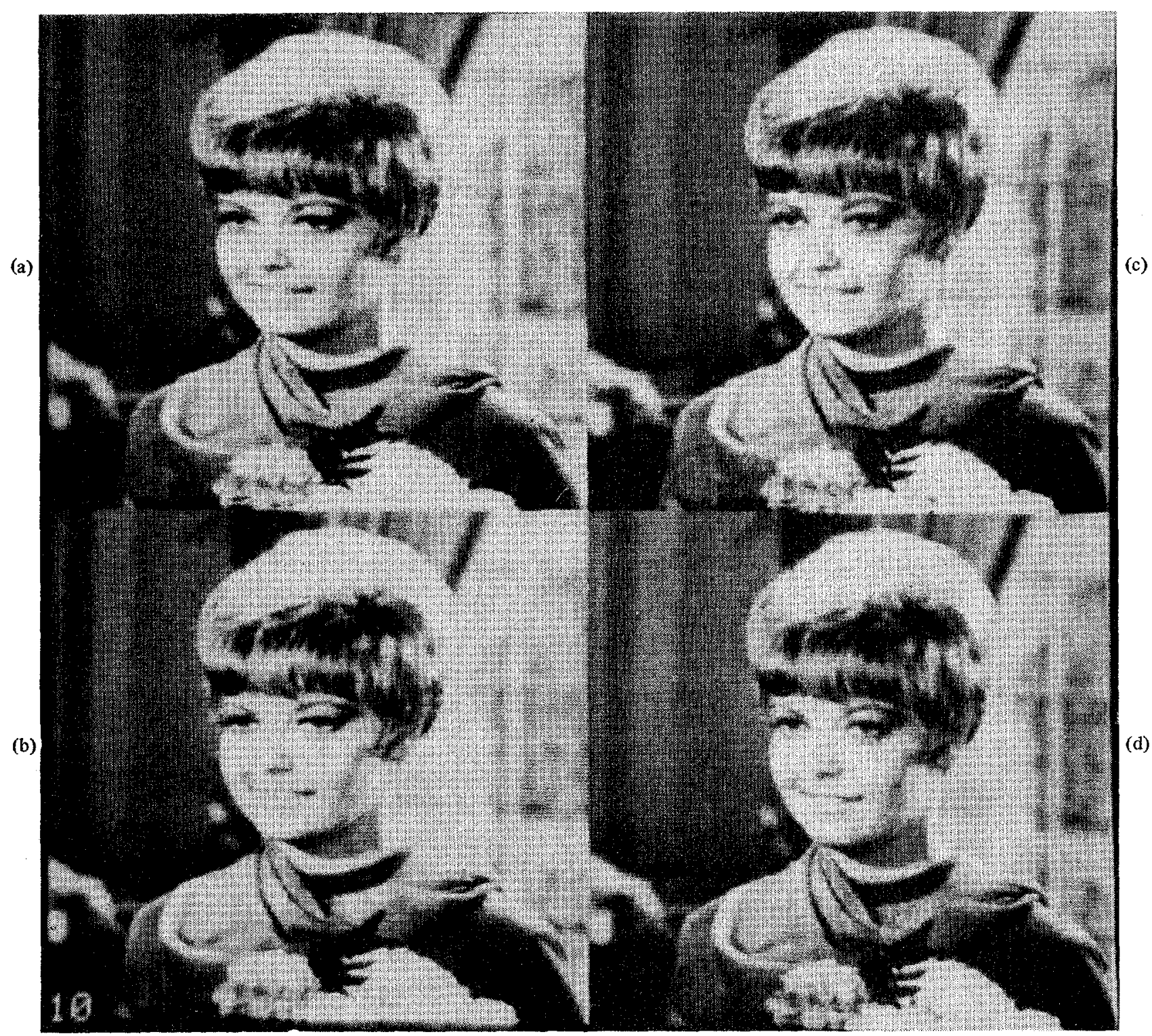

Fig. 17. Reconstructed images from ADPCM of the perceptual visual representation (two-level quantizer, $D=1.5, M=32$ ). (a) $P=3$, $F_{c 0}=$ samples $/^{\circ}$, SNR $=26.7 \mathrm{~dB} . \quad$ (b) $P=8, F_{c 0}=20$ samples $/{ }^{\circ}$, $\mathrm{SNR}=28.2 \mathrm{~dB}$. (c) $P=3, F_{c 0}=10$ samples $/^{\circ}, \mathrm{SNR}=27.2 \mathrm{~dB}$. (d) $P=8, F_{c 0}=10$ samples $/^{\circ}, \mathrm{SNR}=28.1 \mathrm{~dB}$.

scene seems sort of "washed out." These spots may be accounted for by losses in amplitude of the reflectance component because of the center-clipping operation.

Comparison to Plain DPCM: All the previously discussed experimental results referred to ADPCM with both adaptive predictor and adaptive quantization. A natural question to ask at this point is: how much of image quality is sacrificed by using simple DPCM without adapting the predictor and/or the quantizer?

First, we considered the performance of DPCM with only adaptive quantization. The predictor was fixed, and its parameters were obtained by applying 2-D linear prediction to the entire image, which can be considered as a $256 \times 256$ frame. The resulting SNR's were approximately the same (about $1 \mathrm{~dB}$ 


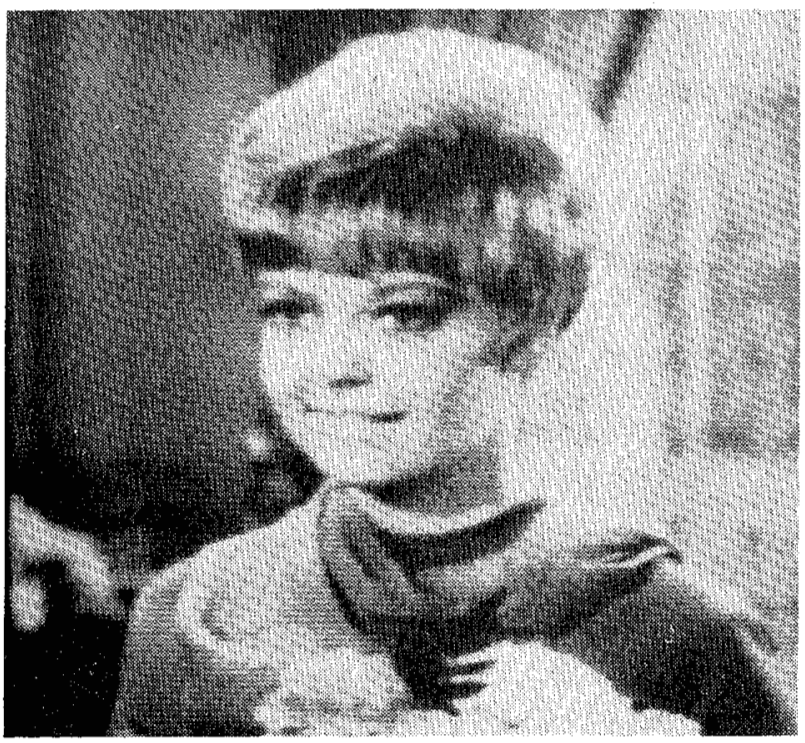

Fig. 18. Reconstructed image from ADPCM of the perceptual visual representation using a three-level quantizer $(K=2, D=2, P=3, M=$ $32, R=0.73 \mathrm{bit} / \mathrm{pel}, \mathrm{SNR}=25.3 \mathrm{~dB}$ ).

lower) as in the case of ADPCM. However, the subjective quality of the DPCM reconstructed images was inferior due to granular noise, which was particularly annoying at bit rates below 1 bit/pixel with a three-level quantizer. Unfortunately, the superior performance of ADPCM over DPCM with only adaptive quantization can be seen only on high-quality image display systems, and it could not be reproduced on printed photographs in this paper. Furthermore, if other than the optimal fixed prediction coefficients were used, both the SNR and the image quality dropped drastically.

Some experiments were also done with a simple DPCM with fixed predictor and a nonadaptive quantizer. The fixed stepsize of the quantizer was proportional to the rms value of the prediction error over the entire image obtained by using the coefficients of the optimum fixed predictor. With a two-level quantizer the resulting reconstructed images had about a $2 \mathrm{~dB}$ lower SNR than for ADPCM and severe granular noise. With a three-level quantizer and bit rates lower than $1 \mathrm{bit} / \mathrm{pixel}$, severe, unacceptable distortions of the scene texture became apparent in the reconstructed image.

\section{CONCLUSIONS}

Two-dimensional linear prediction removes much of the redundancy in monochromatic 2-D image signals. For positive image samples, linear prediction performs better if the bias is subtracted from the samples. The stability of the inverse prediction error filter interacts with the estimation of the optimum bias. Stability can be guaranteed only in the case of 2-D separable linear prediction, which yields approximately the same performance as in the nonseparable case when using the autocorrelation method. Linear prediction yields a smaller normalized prediction error when applied to density images than when applied to intensity images.

Linear prediction of images can be satisfactorily applied to adaptive predictive image coding. By using an ADPCM scheme of the same complexity as the ones used in predictive coding of speech, reconstructed monochrome images of high fidelity can be obtained at an approximate rate of $1 \mathrm{bit} / \mathrm{pixel}$. For bit rates below $1 \mathrm{bit} / \mathrm{pixel}$, a three-level center-clipping quantizer may be used to yield images possessing less granular noise and sharper edges, but with some "contouring" effects, which can be avoided if the coding takes place in the perceptual visual domain (high-pass filtered densities) of the image. In general, good image quality has been obtained at rates as low as 0.7 bits/pixel, which corresponds to a compression factor of 8 to 0.7 or approximately 11 to 1 .

\section{APPENDIX}

Theorem: Consider the first quadrant recursive filter $1 / B\left(z_{1}\right.$, $z_{2}$ ) with

$$
B\left(z_{1}, z_{2}\right)=\sum_{k=0}^{Q} \sum_{l=0}^{R} b(k, l) z_{1}^{-k} z_{2}^{-l}
$$

where $Q, R \geqslant 0, \max (Q, R) \geqslant 1$, and $b(0,0) \neq 0$. This filter is stable only if (necessary condition)

$$
b(0,0) \cdot B(1,1)>0 \text {. }
$$

Proof: Huang's Theorem for stability [9] says that the recursive filter $1 / B\left(z_{1}, z_{2}\right)$ is stable only if

$$
B\left(z_{1}, u\right) \neq 0, \quad\left|z_{1}\right| \geqslant 1 \text {, for ary } u \text { such that }|u| \geqslant 1 .
$$

For $u=1, B\left(z_{1}, u\right)$ reduces to

$$
B\left(z_{1}, 1\right)=z_{1}^{-Q}\left[\sum_{k=0}^{Q} \sum_{i=0}^{R} b(k, l) \quad z_{1}^{Q-k}\right] .
$$

Lemma: The 1-D polynomial $P(x)=a_{0} x^{N}+a_{1} x^{N-1}+\cdots+$ $a_{N}$ with real coefficients has all its roots inside the unit circle only if $a_{0} \cdot P(1)>0$. The proof of this lemma is very easy and therefore will not be given.

To satisfy condition (A3) it is sufficient to require that the 1.D polynomial in $z_{1}$ of degree $Q$ inside the brackets of (A4) have all its roots inside the unit circle. According to the above lemma, this is true only if

$$
B(1,1) \cdot \sum_{l=0}^{R} b(0, l)>0 .
$$

Let us now interchange the roles of $z_{1}$ and $z_{2}$ in (A3) and consider the case $u=\infty$, then

$$
B\left(\infty, z_{2}\right)=z_{2}^{-R}\left[\sum_{l=0}^{R} b(0, l) z_{2}^{R-l}\right] .
$$

Similarly, to satisfy (A3), the roots of the 1-D polynomial in $z_{2}$ inside the brackets of (A6) must be inside the unit circle, which according to the lemma happens only if

$$
b(0,0) \cdot \sum_{l=0}^{R} b(0, l)>0 .
$$

By combining (A5) and (A7), the proof of (A2) is completed. This theorem is stated for the case where $B\left(z_{1}, z_{2}\right)$ has a rectangular $Q \times R$ first quadrant region of support; however, the 
theorem is also true when $B\left(z_{1}, z_{2}\right)$ has an arbitrary first quadrant region of support. In the case where $b(0,0)=1$, condition (A2) reduces to $B(1,1)>0$, to which the necessary condition $(21)$ of Section. II refers.

\section{REFERENCES}

[1] B. S. Atal and S. L. Hanauer, "Speech analysis and synthesis by linear prediction of the speech wave," J. Acoust. Soc. Amer., vol. 50, pp. $637-655,1971$.

[2] B. S. Atal and M. R. Schroeder, "Adaptive predictive coding of speech signals," Bell Syst. Tech. J., vol. 49, pp. 1973-1986, Oct. 1970.

[3] J. Makhoul, "Linear prediction: A tutorial review," Proc. IEEE, vol. $63, \mathrm{pp} .561-580,1975$.

[4] J. D. Markel and A. H. Gray, Jr., Linear Prediction of Speech. New York: Springer-Verlag, 1976.

[5] L. R. Rabiner and R. W. Schafer, Digital Processing of Speech Signals. Englewood Cliffs, NJ: Prentice-Hall, 1978.

[6] A. N. Netravali and J. O. Limb, "Picture coding: A review," Proc. IEEE, pp. 366-406, Mar. 1980.

[7] A. K. Jain, "Image data compression: A review;" Proc. IEEE, pp. 349-389, Mar. 1981.

[8] - "Advances in mathematical models for image processing," Proc. IEEE, pp. 502-528, May 1981.

[9] D. E. Dudgeon and R. M. Mersereau, Multi-Dimensional Digital Signal Processing. Englewood Cliffs, NJ: Prentice-Hall, 1983.

[10] J. H. Justice, "A Levinson-type algorithm for two-dimensional Wiener filtering using bivariate Szego polynomials," Proc. IEEE, pp. 882-886, June 1977.

[11] T. L. Marzetta, "A linear prediction approach to two-dimensional spectral factorization and spectral estimation," Ph.D. dissertation, Massachusetts Inst. Technol., Cambridge, Feb. 1978.

[12] T. Huang, "Stability of two-dimensional recursive filters," IEEE Trans. Audio Electroacoust., pp. 158-163, June 1972.

[13] T. G. Stockham, Jr., "Image processing in the context of a visual model," Proc. IEEE, pp. 828-842, July 1972.

[14] A. V. Oppenheim, R. W. Schafer, and T. G. Stockham, Jr., "Nonlinear filtering of multiplied and convolved signals," Proc. IEEE, pp. 1264-1291, Aug. 1968.

[15] B. S. Atal, "Predictive coding of speech at low bit rates," IEEE Trans. Commun., vol. COM-30, pp.600-614, Apr. 1982.

[16] B.S. Atal and M. R. Schroeder, "Improved quantizer for adaptive predictive coding for speech signals at low bit rates," in Proc. 1980 Int. Conf. Acoust., Speech, Signal Processing, Denver, CO, pp. 535-538.

[17] L. H. Zetterberg, S. Ericsson, and H. Brusewitz, "Interframe DPCM with adaptive quantization and entropy coding," IEEE Trans. Commun., vol. COM-30, pp. 1888-1899, Aug. 1982.

[18] P. A. Maragos, "On the adaptive predictive coding of digital monochrome still images," Master's thesis, Georgia Inst. Technol., A tlanta, June 1982.

[19] R. G. Gallagher, Information Theory and Retiable Communication. New York: Wiley, 1968.

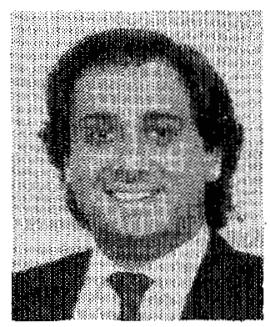

Petros A. Maragos ( $\mathrm{S} 84$ ) was born in Kalymnos, Greece, on November 4, 1957. He received the Diploma in electrical engineering from the $\mathrm{Na}$ tional Technical University of Athens, Athens, Greece, in 1980 and the M.S. degree in electrical engineering from the Georgia Institute of Technology, Atlanta, in 1982.

Since 1980 he has been working as a Research Assistant at the Digital Signal Processing Laboratory of the Electrical Engineering School at Georgia Tech, where he is currently pursuing the
Ph.D. degree in the area of digital image processing. His research interests include the general area of digital signal processing with emphasis on image processing, as well as digital communications and pattern recognition.

$\mathrm{Mr}$. Maragos is a member of the Technical Chamber of Commerce of Greece.

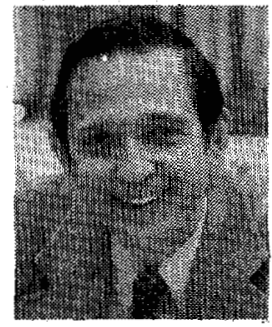

Ronald W. Schafer (S'62-M'67-SM'74-F'77) received the B.S.E.E. and M.S.E.E. degrees from the University of Nebraska, Lincoln, in 1961 and 1962, respectively, and the Ph.D. degree in electrical engineering from the Massachusetts Institute of Technology, Cambridge, in 1968.

From 1968 to 1974 he was a member of the Acoustics Research Department at Bell Laboratories, Murray Hill, NJ, where he was engaged in research on speech analysis and synthesis, digital signal processing techniques, and digital waveform coding. Since 1974 he has been on the faculty of Georgia Institute of Technology, Atlanta, as John O. McCarty/Audichron Professor of Electrical Engineering. He is coauthor of the textbooks, Digital Signal Processing and Digital Processing of Speech Signals.

Dr. Schafer has served as Associate Editor of the IEEE TRANSACTIONS on ACoustics, Speech, AND Signal Processing. He has also been a member of the Digital Signal Processing and Speech Processing Committees of the IEEE Acoustics, Speech, and Signal Processing Society and has served as President of that Society. He is a Fellow of the Acoustical Society of America and a member of Sigma Xi and Eta Kappa Nu. He shared the 1980 Emanuel R. Piore Award with L. R. Rabiner, and he was awarded the ASSP Achievement Award in 1979 and the Society Award in 1983.

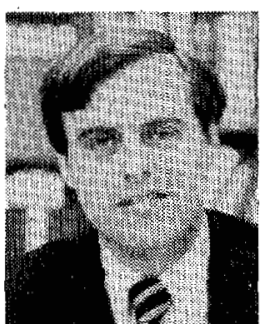

Russell M. Mersereau (S'69-M'73-SM'78-F'83) was born in Cambridge, MA, on August 29, 1946. He received the S.B., S.M., and Sc.D. dégrees from the Massachusetts Institute of Technology, Cambridge, in 1969,1969 , and 1973 , respectively.

From 1971 to 1973 he was an Instructor in the Department of Electrical Engineering at M.1.T. and from 1973 to 1975 he was with the Research Laboratory of Electronics and Department of Electrical Engineering as a Research Associate. Currently he is a Professor in the School of Electrical Engineering, Georgia Institute of Technology, Atlanta, where his primary research interest is multidimensional signal processing.

Dr. Mersereau was formerly the Associate Editor for Signal Processing of the IEEE Transactions on Acoustics, Speech, and Signal ProcessING and Technical Chairman of that Society's International Conference in 1981. He is a former member of the ASSP AdCom and the Steering Committee for the TRANSACTIONS ON MEDICAL IMAGiNG, and currently is a member of the Multidimensional Digital Signal Processing Committee of ASSP and the Editorial Board of the Proceedings of THe IEEE. He is the corecipient with D. E. Dudgeon of the 1976 Browder J. Thompson Memorial Prize from the IEEE for the best paper by an author under the age of 30 and a recipient of the 1976 Research Unit Award from the Southeastern Section of the ASEE. He received a teaching award from the School of Electrical Engineering at the Georgia Institute of Technology in 1978. 